\title{
Homogeneous quaternionic Kähler structures on eight-dimensional non-compact quaternion-Kähler symmetric spaces *
}

\author{
M. Castrillón López, P. M. Gadea, and J. A. Oubiña
}

\begin{abstract}
For each non-compact quaternion-Kähler symmetric space of dimension 8, all of its descriptions as a homogeneous Riemannian space (obtained through the Witte's refined Langlands decomposition) and the associated homogeneous quaternionic Kähler structures are studied.
\end{abstract}

\section{Introduction and Preliminaries}

Introduction. As it is well-known, quaternion-Kähler symmetric spaces were classified by Wolf [21, Th. 5.4]. The non-compact ones are moreover a particular case of Alekseevsky spaces ([1, p. 338], [8, Th. 2.28]). On the other hand, a theoretical-representation classification of homogeneous quaternionic Kähler structures was given by Fino [9, L. 5.1]. Then, a classification by real tensors was obtained in [6, Th. 3.15], where furthermore a characterization of the quaternionic hyperbolic space in terms of such structures was given.

The aim of this paper is to find all the homogeneous Riemannian structures associated to every homogeneous description (obtained through Witte's refined Langlands decomposition [20, Th. 1.2]) of each 8-dimensional non-compact quaternion-Kähler symmetric space, and to determine their types as homogeneous quaternionic Kähler structures.

Non-compact quaternion-Kähler spaces are found in the formulation of the coupling of matter fields in $N=2$ supergravity as target spaces of some nonlinear sigma models (Bagger-Witten [4, p. 7], Cecotti [7, p. 32], de Wit-Van Proeyen [19, p. 26]; see also Fré et al. [10, p. 65] and references therein). Since Fino's classification is natural, it seems reasonable the existence of links between the classification of homogeneous quaternionic Kähler structures and a possible

\footnotetext{
* Partially supported by DGICYT, Spain, under Grant MTM2005-00173.

2000 AMS Subject Classification: Primary 53c26; Secondary 53c3o, 53c 35

Key words: non-compact quaternion-Kähler symmetric spaces, homogeneous Riemannian structures, homogeneous quaternionic Kähler structures, parabolic subgroups, refined Langlands decomposition.
} 
classification of some physical structures and models. This would, in a sense, be similar to a recent result by Meessen [16, Th. 2]: A connected homogeneous Lorentzian space admits a non-vanishing degenerate homogeneous Lorentzian structure in Tricerri-Vanhecke's class $\mathcal{T}_{1} \oplus \mathcal{T}_{3}$ (see [18, p. 41], [11, Th. 2.1], [17, p. 562]) if and only if it is a singular homogeneous plane wave. Moreover, we give in the present paper some candidates to such target manifolds.

As for the contents, we first establish (Theorem 2.3) a result related to Witte's Theorem on closed co-compact subgroups of a semisimple Lie group with finite center ([20, Th. 1.2]), which gives necessary and sufficient conditions for a connected closed co-compact subgroup of such a group to act transitively (see also Gordon-Wilson [12, Th. 6.9]). We then furnish explicitly (Proposition 2.4 ), for the present case of symmetric spaces, the coefficients appearing in the symmetries of homogeneous structures characteristic of the quaternionic Kähler case (that is, equations (1.3)). Further, for each one of spaces under study, i.e. the complex hyperbolic Grassmannian $S U(2,2) / S(U(2) \times U(2))$, the quaternionic hyperbolic space $\mathbb{H H}(2)=S p(2,1) /(S p(2) \times S p(1))$, and the exceptional space $G_{2(2)} / S O(4)$, we first give the corresponding quaternionic structure. This is associated to a natural structure of quaternion-Hermitian vector space on the Lie algebra $\mathfrak{a}+\mathfrak{n}$ of the solvable factor $A N$ of an Iwasawa decomposition of its respective full connected group of isometries. According to Alekseevsky [1, Th. 1.1] (see also Heber [13, Cor. 5.5]), its metric is unique up to isometry and scaling.

Then, we obtain all the descriptions of each space and the corresponding homogeneous quaternionic Kähler structures. These structures are associated to the reductive decompositions defined via the Witte's refined Langlands decomposition of the identity component of each parabolic subgroup of the full connected isometry group. It turns out that each parabolic subgroup provides a homogeneous description which, although is the simplest possible one, suffices to obtain all such homogeneous structures. We exhibit the corresponding structures in Propositions 3.1, 3.2, 3.3, and their types in Theorem 3.4.

We have studied the twelve-dimensional case in [5].

Homogeneous quaternionic Kähler structures. Let $(M, g)$ be a connected, simply-connected, and complete Riemannian manifold. Ambrose and Singer [3, p. 656] gave a characterisation for $(M, g)$ to be homogeneous in terms of a $(1,2)$ tensor field $S$, usually called (Tricerri-Vanhecke [18, Def. 2.1]) a homogeneous Riemannian structure: Let $\nabla$ be the Levi-Civita connection of $g$ and $R$ its curvature tensor. Then the manifold is homogeneous Riemannian if and only if there exists a $(1,2)$ tensor field $S$ such that the Ambrose-Singer equations

$$
\widetilde{\nabla} g=0, \quad \widetilde{\nabla} R=0, \quad \widetilde{\nabla} S=0,
$$

where $\widetilde{\nabla}=\nabla-S$, are satisfied.

Let $(M, g, v)$ be a quaternion-Kähler manifold, $v$ denoting the distinguished rank-three subbundle of the bundle of $(1,1)$ tensor fields on $M$. Then $(M, g, v)$ is said to be a homogeneous quaternion-Kähler space if it admits a transitive 
group of isometries (Alekseevsky-Cortés [2, Th. 1.1]). The canonical 4 -form $\Omega$ is known to be globally defined and we have

Corollary 1.1. (To KIRIČENKO [14, Th. 1]) A connected, simply-connected, and complete quaternion-Kähler manifold $(M, g, v)$ is homogeneous if and only if there exists a tensor field $S$ of type $(1,2)$ on $M$ satisfying

$$
\widetilde{\nabla} g=0, \quad \widetilde{\nabla} R=0, \quad \widetilde{\nabla} S=0, \quad \widetilde{\nabla} \Omega=0,
$$

where $\widetilde{\nabla}=\nabla-S$.

Such a tensor $S$ is called a homogeneous quaternionic Kähler structure on $M$. The condition $\widetilde{\nabla} \Omega=0$ can be replaced by the set of equations

$$
\begin{aligned}
& S_{X} J_{1}=\theta^{3}(X) J_{2}-\theta^{2}(X) J_{3}, \\
& S_{X} J_{2}=\theta^{1}(X) J_{3}-\theta^{3}(X) J_{1}, \\
& S_{X} J_{3}=\theta^{2}(X) J_{1}-\theta^{1}(X) J_{2},
\end{aligned}
$$

for certain differential 1-forms $\theta^{a}, a=1,2,3$, where $\left\{J_{1}, J_{2}, J_{3}\right\}$ is a local basis of $v$ satisfying the conditions $J_{a}^{2}=-I, J_{a} J_{b}=-J_{b} J_{a}=J_{c}$, for each cyclic permutation $(a, b, c)$ of $(1,2,3)$. Denoting $S_{X Y Z}=g\left(S_{X} Y, Z\right)$, one has

$$
S_{X J_{1} Y J_{1} Z}-S_{X Y Z}=\theta^{3}(X) g\left(J_{2} Y, J_{1} Z\right)-\theta^{2}(X) g\left(J_{3} Y, J_{1} Z\right),
$$

and the two other similar equations.

Note that the conditions (1.4) (equivalently, equations (1.3)), besides the general condition $S_{X Y Z}=-S_{X Z Y}$, are the symmetries satisfied by a homogeneous quaternionic Kähler structure $S$.

A quaternion-Hermitian real vector space $\left(V,\langle\rangle,, J_{1}, J_{2}, J_{3}\right)$ is the model for the tangent space at any point of a quaternion-Kähler manifold. Consider the space of tensors $\mathcal{T}(V)=\left\{S \in \otimes^{3} V^{*}: S_{X Y Z}=-S_{X Z Y}\right\}$ and its vector subspace $\mathcal{V}$ of tensors satisfying (1.4) with $\langle\rangle,, \theta^{a} \in V^{*}$. Then $\mathcal{V}=\check{\mathcal{V}}+\hat{\mathcal{V}}$, where

$$
\begin{gathered}
\check{\mathcal{V}}=\left\{\Theta \in \otimes^{3} V^{*}: \Theta_{X Y Z}=\sum_{a=1}^{3} \theta^{a}(X)\left\langle J_{a} Y, Z\right\rangle, \theta^{a} \in V^{*}\right\}, \\
\hat{\mathcal{V}}=\left\{T \in \otimes^{3} V^{*}: T_{X Y Z}=-T_{X Z Y}, T_{X J_{a} Y J_{a} Z}=T_{X Y Z}, a=1,2,3\right\} .
\end{gathered}
$$

The decomposition above of $\mathcal{V}$ is orthogonal with respect to the scalar pro$\operatorname{duct}\left(\right.$, ) defined by $\left(S, S^{\prime}\right)=\sum_{r, s, t=1}^{4 n} S_{e_{r} e_{s} e_{t}} S_{e_{r} e_{s} e_{t}}^{\prime}$, where $\left\{e_{r}\right\}_{r=1, \ldots, 4 n}$ is an orthonormal basis of $V$. Then $\check{\mathcal{V}}$ and $\hat{\mathcal{V}}$ decompose into two and three subspaces, respectively, giving an orthonormal sum of five subspaces which are invariant and irreducible under the action of $S p(n) S p(1)$, due to the following theorem. Let $E$ denote the standard representation of $S p(n)$ on $\mathbb{C}^{2 n} ; S^{3} E$ the 3 -symmetric product of $E ; K$ the irreducible $S p(n)$-module of highest weight $(2,1,0, \ldots, 0)$; $H$ the standard representation of $S p(1)$ on $\mathbb{C}^{2}$; and $S^{3} H$ the 4-dimensional symmetric product of $H$. Using brackets for real representations and with the usual notations we have 
Theorem 1.2. (FInO $[9$, L. 5.4]) The space $[E H] \otimes(\mathfrak{s p}(1) \oplus \mathfrak{s p}(n))$ of homogeneous quaternionic Kähler structures splits into invariant and irreducible subspaces under the action of $S p(n) S p(1)$ as $[E H]+\left[E S^{3} H\right]+[E H]+\left[S^{3} E H\right]+$ $[K H]$.

Let $\mathcal{Q} \mathcal{K}_{i}$ be the $i$ th summand in Fino's classification. Then we have

Theorem 1.3. ([6, Th. 3.15]) If $n \geqslant 2$, then $\mathcal{V}$ decomposes into the direct sum of the following subspaces invariant and irreducible under the action of $S p(n) S p(1)$ :

$$
\begin{aligned}
& \mathcal{Q K}_{1}=\left\{\Theta \in \check{\mathcal{V}}: \Theta_{X Y Z}=\sum_{a=1}^{3} \theta\left(J_{a} X\right)\left\langle J_{a} Y, Z\right\rangle, \theta \in V^{*}\right\}, \\
& \mathcal{Q K}_{2}=\left\{\Theta \in \check{\mathcal{V}}: \Theta_{X Y Z}=\sum_{a=1}^{3} \theta^{a}(X)\left\langle J_{a} Y, Z\right\rangle, \sum_{a=1}^{3} \theta^{a} \circ J_{a}=0, \theta^{a} \in V^{*}\right\}, \\
& \mathcal{Q K}_{3}=\left\{T \in \hat{\mathcal{V}}: T_{X Y Z}=\langle X, Y\rangle \vartheta(Z)-\langle X, Z\rangle \vartheta(Y)\right. \\
& \left.+\sum_{a=1}^{3}\left(\left\langle X, J_{a} Y\right\rangle \vartheta\left(J_{a} Z\right)-\left\langle X, J_{a} Z\right\rangle \vartheta\left(J_{a} Y\right)\right), \vartheta \in V^{*}\right\}, \\
& \mathcal{Q K}_{4}=\left\{T \in \hat{\mathcal{V}}: T_{X Y Z}=\frac{1}{2}\left(T_{Y Z X}-T_{Z X Y}\right.\right. \\
& \left.\left.+\sum_{a=1}^{3}\left(T_{J_{a} Y J_{a} Z X}-T_{J_{a} Z J_{a} Y X}\right)\right), c_{12}(T)=0\right\}, \\
& \mathcal{Q} \mathcal{K}_{5}=\left\{T \in \hat{\mathcal{V}}: T_{X Y Z}=-\frac{1}{4}\left(T_{Y Z X}-T_{Z Y X}+\sum_{a=1}^{3}\left(T_{J_{a} Y J_{a} Z X}-T_{J_{a} Z J_{a} Y X}\right)\right)\right\} .
\end{aligned}
$$

We will denote the sum of classes $\mathcal{Q} \mathcal{K}_{i}+\mathcal{Q K} \mathcal{K}_{j}$ by $\mathcal{Q} \mathcal{K}_{i j}$, and so on. In particular, we have $\check{\mathcal{V}}=\mathcal{Q} \mathcal{K}_{12}, \hat{\mathcal{V}}=\mathcal{Q K}_{345}$.

Remark 1.4. Let $(M, g, v)$ be a homogeneous quaternion-Kähler space. For any given $p \in M$, the decomposition of $\mathcal{V}_{p}$ given by Theorem 1.3 depends only on $v_{p}$ and not on the chosen bases $\left\{\left(J_{1}\right)_{p},\left(J_{2}\right)_{p},\left(J_{3}\right)_{p}\right\}$, so the irreducible summands $\left.(\mathcal{Q K})_{i}\right)_{p}$ give well-defined bundles $\mathcal{Q} \mathcal{K}_{i}$ over $M$. Moreover, if $S$ is a homogeneous quaternionic Kähler structure and $S_{p}$ belongs to a given invariant subspace of $\mathcal{V}_{p}$, then $S_{q}$ belongs to the invariant subspace of $\mathcal{V}_{q}$ of the same type at any other $q \in M$, and $S$ is a section of the corresponding vector bundle.

\section{Non-compact homogeneous spaces}

Non-discrete co-compact subgroups acting transitively. Gordon and Wilson gave in [12, Th. 6.9] a theorem of characterization of the isometry groups acting transitively on non-compact Riemannian symmetric spaces. We will prove Theorem 2.3, related to Witte's Theorem 2.2, which suffices for our purposes.

Let $(M, g)$ be a connected non-compact Riemannian manifold and $G$ a Lie group of isometries acting transitively and effectively on $M$. If $K$ is the isotropy group at any fixed point $o \in M$, then $M=G / K$. We look for the connected closed subgroups $\hat{G}$ of $G$ acting transitively by isometries on $M$. Denoting $K_{\hat{G}}=\hat{G} \cap K$, one must have $M \equiv \hat{G} / K_{\hat{G}}$, and thus other possible description of $(M, g)$ as a homogeneous Riemannian space. Note that since $G$ acts effectively then $K$ is compact, and if $G=\hat{G} K$ then $G / \hat{G} \equiv K / K_{\hat{G}}$. Then we have 
Lemma 2.1. (1) A subgroup $\hat{G}$ of $G$ acts transitively on $M$ if and only if $G=\hat{G} K$. (2) If $\hat{G}$ is a closed subgroup of $G$ which acts transitively on $M$, then it is co-compact, that is, $G / \hat{G}$ is compact.

The structure of the non-discrete co-compact subgroups of a connected semisimple Lie group $G$ with finite center was given in Witte [20, Th. 1.2]: Let $\mathfrak{g}$ be the Lie algebra of $G, \mathfrak{a}$ a maximal $\mathbb{R}$-diagonalizable subalgebra of $\mathfrak{g}, \Sigma$ the set of roots of $(\mathfrak{g}, \mathfrak{a})$, and $\mathfrak{g}=\mathfrak{g}_{0}+\sum_{f \in \Sigma} \mathfrak{g}_{f}$ the restricted-root space decomposition, with $\mathfrak{g}_{0}=\mathfrak{a}+Z_{\mathfrak{k}}(\mathfrak{a})$, where $Z_{\mathfrak{k}}(\mathfrak{a})$ stands for the centralizer of $\mathfrak{a}$ in $\mathfrak{k}$. Write $\Sigma^{+}$for the set of positive roots with respect to a certain notion of positivity for $\mathfrak{a}^{*}$, and let $\Pi$ be the set of simple restricted roots. For each subset $\Psi$ of $\Pi$, let $[\Psi]$ be the set of restricted roots that are linear combinations of elements of $\Psi$. Then, the (connected) standard parabolic subgroup $P_{\Psi}^{0}$ is defined as the connected subgroup of $G$ having Lie algebra $\mathfrak{p}_{\Psi}=\mathfrak{g}_{0}+\sum_{f \in \Sigma^{+} \cup[\Psi]} \mathfrak{g}_{f}=\mathfrak{l}^{\prime}+\mathfrak{n}_{\Psi}$, where $\mathfrak{l}^{\prime}=\mathfrak{g}_{0}+\sum_{f \in[\Psi]} \mathfrak{g}_{f}=\mathfrak{l}_{\Psi}^{\prime}+\mathfrak{e}_{\Psi}^{\prime}+\mathfrak{a}_{\Psi}$, with $\mathfrak{l}_{\Psi}^{\prime}$ semisimple with non-compact summands, $\mathfrak{e}_{\Psi}^{\prime}$ compact reductive, $\mathfrak{a}_{\Psi}$ the non-compact part of the center of $\mathfrak{l}^{\prime}$, and with $\mathfrak{n}_{\Psi}=\sum_{f \in \Sigma+\backslash[\Psi]} \mathfrak{g}_{f}$ nilpotent. On the Lie group level we have [20, Th. 1.2] the refined Langlands decomposition $P_{\Psi}^{0}=L_{\Psi}^{\prime} E_{\Psi}^{\prime} A_{\Psi} N_{\Psi}$, and one has the

Theorem 2.2. (WITTE [20, Th. 1.2]) Let $L$ be a connected normal subgroup of $L_{\Psi}^{\prime}$ and $E$ a connected closed subgroup of $E_{\Psi}^{\prime} A_{\Psi}$. Then there is a closed cocompact subgroup $\hat{G}$ of $G$ contained in $P_{\Psi}$ with identity component $\hat{G}^{0}=L E N_{\Psi}$. Further, every closed co-compact subgroup of $G$ arises in this way.

If $I_{0}(M)$ is the full connected isometry group of $M$, to find the homogeneous descriptions of the non-compact Riemannian symmetric space $(M, g)$, we will look among the co-compact subgroups $\hat{G}$ of $I_{0}(M)$ given by Witte's Theorem 2.2 for those connected groups acting transitively. Note that if a Lie group acts transitively on $M$, so does its identity component. From Lemma 2.1 and Theorem 2.2, and writing $N=N_{\emptyset}$ when $\Psi=\emptyset \subset \Pi$, we have

Theorem 2.3. Let $G$ be a connected semisimple Lie group with finite center and $G=K A N$ the Iwasawa decomposition. A connected closed co-compact subgroup $\hat{G}=L E N_{\Psi}$ of $G$ acts transitively on $M=G / K$ if and only if: (a) The projections of the Lie algebra $\mathfrak{l} \subset \mathfrak{l}^{\prime}=\mathfrak{g}_{0}+\sum_{f \in[\Psi]} \mathfrak{g}_{f}$ of $L$ to $\sum_{f \in \Sigma^{+} \cap[\Psi]} \mathfrak{g}_{f}$ and to $\mathfrak{a}_{\Psi}^{\perp}$ are surjective, $\mathfrak{a}_{\Psi}^{\perp}$ being the orthogonal complement to $\mathfrak{a}_{\Psi}$ in $\mathfrak{a} \subset \mathfrak{g}_{0}=$ $\mathfrak{a}_{\Psi}+\mathfrak{a}_{\Psi}^{\perp}+Z_{\mathfrak{k}}(\mathfrak{a})$. (b) The projection of the Lie algebra $\mathfrak{e} \subset \mathfrak{e}_{\Psi}^{\prime}+\mathfrak{a}_{\Psi}$ of $E$ to $\mathfrak{a}_{\Psi}$ is surjective.

Proof. Transitivity is equivalent to $\hat{G} K=G$. Since $\hat{G}$ and $K$ are connected subgroups of $G$, this is equivalent to saying that the Lie algebra $\hat{\mathfrak{g}}+\mathfrak{k}$ of $\hat{G} K$ coincides with $\mathfrak{g}$. On the other hand, the Iwasawa decomposition of $G$ gives us the vector space direct sum decomposition $\mathfrak{g}=\mathfrak{k}+\mathfrak{a}+\mathfrak{n}$. Then, if $\pi_{\mathfrak{k}}, \pi_{\mathfrak{a}}$, and $\pi_{\mathfrak{n}}$ denote the projections to the summands $\mathfrak{k}, \mathfrak{a}$ and $\mathfrak{n}$, a necessary and sufficient condition for $\hat{\mathfrak{g}}+\mathfrak{k}=\mathfrak{g}$ to be fulfilled, is to have $\pi_{\mathfrak{a}}(\hat{\mathfrak{g}})=\mathfrak{a}$ and $\pi_{\mathfrak{n}}(\hat{\mathfrak{g}})=\mathfrak{n}$. Hence we have that: (1) Since $\mathfrak{n}=\sum_{f \in \Sigma^{+}} \mathfrak{g}_{f}$ and $\mathfrak{n}_{\Psi}=\sum_{f \in \Sigma^{+} \backslash[\Psi]} \mathfrak{g}_{f}$, to get $\pi_{\mathfrak{n}}(\hat{\mathfrak{g}})=\mathfrak{n}$, the first condition in (a) must be true, and (2) To have $\pi_{\mathfrak{a}}(\hat{\mathfrak{g}})=\mathfrak{a}$, 
as on the one hand $E$ is a subgroup of $E_{\Psi}^{\prime} A_{\Psi}$ and on the other hand $\mathfrak{a}_{\Psi} \subset \mathfrak{a}$, the other condition in (a) and the one in (b) must be satisfied.

Homogeneous Riemannian structures. Let $(M, g, v)$ a connected simplyconnected homogeneous quaternion-Kähler space. We have $M=G / K$, where $G=I_{0}(M)$ and $K$ is the isotropy subgroup of $G$ at a point $o \in M$. Let $\hat{G}$ be a connected closed Lie subgroup of $G$ which acts transitively on $M$. The isotropy group of this action at $o=K \in M$ is $H=K_{\hat{G}}=\hat{G} \cap K$. Then $M=G / K$ has also the description $M \equiv \hat{G} / H$, and $o \equiv H \in \hat{G} / H$.

Consider a reductive decomposition of the Lie algebra $\hat{\mathfrak{g}}$ of $\hat{G}$, that is, a vector space direct sum $\hat{\mathfrak{g}}=\mathfrak{h}+\mathfrak{m}$, where $\mathfrak{h}$ is the Lie algebra of $H$ and $\operatorname{Ad}(H) \mathfrak{m} \subset \mathfrak{m}$. Since $H$ is connected, the condition $\operatorname{Ad}(H) \mathfrak{m} \subset \mathfrak{m}$ is equivalent to $[\mathfrak{h}, \mathfrak{m}] \subset \mathfrak{m}$. For each $X$ in the Lie algebra $\mathfrak{g}$ of $G$, denote by $X^{*}$ the Killing vector field on $M$ generated by the one-parameter subgroup $\{\exp t X\}$ of $G$ acting on $M$. The vector subspace $\mathfrak{m}$ of $\hat{\mathfrak{g}} \subset \mathfrak{g}$ is identified with the tangent space $T_{o}(M)$ by the isomorphism $X \in \mathfrak{m} \rightarrow X_{o}^{*} \in T_{o}(M)$. The reductive decomposition $\hat{\mathfrak{g}}=\mathfrak{h}+\mathfrak{m}$ defines the homogeneous Riemannian structure $S=\nabla-\widetilde{\nabla}$, where $\widetilde{\nabla}$ is the canonical connection of $M=\hat{G} / H$ with respect to $\hat{\mathfrak{g}}=\mathfrak{h}+\mathfrak{m}$. The connection $\widetilde{\nabla}$ is invariant under the action of $\hat{G}$, it is determined by $\left(\widetilde{\nabla}_{X^{*}} Y^{*}\right)_{o}=\left[X^{*}, Y^{*}\right]_{o}=$ $-[X, Y]_{o}^{*}$, for $X, Y \in \mathfrak{m}$, and the Ambrose-Singer equations (1.1) are satisfied. The tensor field $S$ is also uniquely determined by its value at $o$ because $M \equiv$ $\hat{G} / H$ and $S$ is $\hat{G}$-invariant. We have

$$
\left(S_{X^{*}} Y^{*}\right)_{o}=\left(\nabla_{X^{*}} Y^{*}\right)_{o}+[X, Y]_{o}^{*}=\nabla_{Y_{o}^{*}} X^{*}, \quad X, Y \in \mathfrak{m}
$$

Moreover, as $\Omega$ is $\hat{G}$-invariant, from [15, Ch. 10, Prop. 2.7] it follows that $\widetilde{\nabla} \Omega=$ 0 , so equations (1.2) are satisfied and $S$ is also a homogeneous quaternionic Kähler structure.

Now, suppose that $(M=G / K, g, v)$ is a connected non-compact quaternionKähler symmetric space, and let $\hat{\mathfrak{g}}=\mathfrak{h}+\mathfrak{m}$ be a reductive decomposition corresponding to $M \equiv \hat{G} / H$ as above. We consider a Cartan decomposition $\mathfrak{g}=\mathfrak{k}+\mathfrak{p}$ of the Lie algebra $\mathfrak{g}$ of $G$, and the Iwasawa decomposition $\mathfrak{g}=\mathfrak{k}+\mathfrak{a}+\mathfrak{n}$, where $\mathfrak{k}$ is the Lie algebra of $K, \mathfrak{a} \subset \mathfrak{p}$ is a maximal $\mathbb{R}$-diagonalizable subalgebra of $\mathfrak{g}$, and $\mathfrak{n}$ is a nilpotent subalgebra. Let $A$ and $N$ be the connected abelian and nilpotent Lie subgroups of $G$ whose Lie algebras are $\mathfrak{a}$ and $\mathfrak{n}$, respectively. The solvable Lie group $A N$ acts simply transitively on $M$, so $M$ is isometric to $A N$ equipped with the left-invariant Riemannian metric defined by the scalar product induced on $\mathfrak{a}+\mathfrak{n} \cong \mathfrak{g} / \mathfrak{k} \cong \mathfrak{p}$ by a positive multiple of $B_{\mid \mathfrak{p} \times \mathfrak{p}}$, where $B$ is the Killing form of $\mathfrak{g}$.

If $X \in \mathfrak{g}=\mathfrak{k}+\mathfrak{p}$, we write $X=X_{\mathfrak{k}}+X_{\mathfrak{p}},\left(X_{\mathfrak{k}} \in \mathfrak{k}, X_{\mathfrak{p}} \in \mathfrak{p}\right)$. We have the isomorphisms of vector spaces

$$
\mathfrak{p} \cong \mathfrak{g} / \mathfrak{k} \cong \hat{\mathfrak{g}} / \mathfrak{h} \cong \mathfrak{m} \cong T_{o}(M) \cong \mathfrak{a}+\mathfrak{n},
$$

with

$$
\xi: \mathfrak{p} \stackrel{\cong}{\rightrightarrows}, \quad \mu: \mathfrak{m} \cong T_{o}(M), \quad \zeta: T_{o}(M) \cong \mathfrak{a}+\mathfrak{n},
$$


such that

$$
\xi^{-1}(Z)=Z_{\mathfrak{p}}, \quad \mu(Z)=Z_{o}^{*}, \quad \zeta^{-1}(X)=X_{o}^{*}, \quad Z \in \mathfrak{m}, X \in \mathfrak{a}+\mathfrak{n} .
$$

For each $X \in \mathfrak{g}$, we have $\left(X_{\mathfrak{k}}\right)_{o}^{*}=0$ and $\left(\nabla\left(X_{\mathfrak{p}}\right)^{*}\right)_{o}=0$, and since the Levi-Civita connection $\nabla$ has no torsion, for each $X, Y \in \mathfrak{g}$, we have

$$
\left(\nabla_{X^{*}} Y^{*}\right)_{o}=\left(\nabla_{\left(X_{\mathfrak{p}}\right)^{*}}\left(Y_{\mathfrak{k}}\right)^{*}\right)_{o}=\left[\left(X_{\mathfrak{p}}\right)^{*},\left(Y_{\mathfrak{k}}\right)^{*}\right]_{o}=-\left[X_{\mathfrak{p}}, Y_{\mathfrak{k}}\right]_{o}^{*} .
$$

By (2.1) and (2.3), the homogeneous Riemannian structure $S$ associated to a reductive decomposition $\hat{\mathfrak{g}}=\mathfrak{h}+\mathfrak{m}$ is given by

$$
S_{X_{o}^{*}} Y_{o}^{*}=\left[X_{\mathfrak{k}}, Y_{\mathfrak{p}}\right]_{o}^{*}, \quad X, Y \in \mathfrak{m} .
$$

Then, for each $X, Y \in \mathfrak{a}+\mathfrak{n}$, we have

$$
S_{X_{o}^{*}} Y_{o}^{*}=S_{\xi\left(X_{\mathfrak{p}}\right)_{o}^{*}} \xi\left(Y_{\mathfrak{p}}\right)_{o}^{*}=\left[\left(\xi\left(X_{\mathfrak{p}}\right)\right)_{\mathfrak{k}}, Y_{\mathfrak{p}}\right]_{o}^{*}
$$

This last formula will be helpful to give directly the associated homogeneous Riemannian structure $S$ in terms of a basis of $\mathfrak{a}+\mathfrak{n}$. Moreover, we will get formula (2.6), which furnishes explicitly the coefficients $\theta^{a}, a=1,2,3$, in equations (1.3), for the quaternion-Hermitian structure on $M$ which we will now describe. Before giving it, we note that formulas (2.4) and (2.6) will be useful in the next sections to find the homogeneous Riemannian structures on the spaces under study and their types as homogeneous quaternionic Kähler structures, respectively.

The quaternionic structure on $M$ is defined by a 3 -dimensional ideal $\mathfrak{u} \cong$ $\mathfrak{s p}(1)$ of $\mathfrak{k}$, and we can suppose that $\mathfrak{u}=\left\langle E_{1}, E_{2}, E_{3}\right\rangle$, with

$$
\left[E_{1}, E_{2}\right]=2 E_{3}, \quad\left[E_{2}, E_{3}\right]=2 E_{1}, \quad\left[E_{3}, E_{1}\right]=2 E_{2} .
$$

The next commutative diagram defines the complex structures $J_{a} \in \operatorname{End}(\mathfrak{a}+\mathfrak{n})$ $(1 \leqslant a \leqslant 3)$, which make $\left(\mathfrak{a}+\mathfrak{n},\langle\rangle,, J_{1}, J_{2}, J_{3}\right)$ a quaternion-Hermitian vector space isomorphic to $\left(T_{o}(M), g_{o},\left(J_{1}\right)_{o},\left(J_{2}\right)_{o},\left(J_{3}\right)_{o}\right)$.

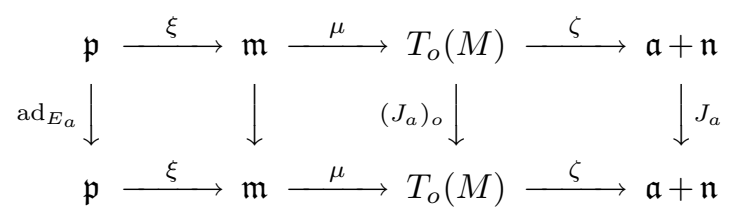

Now, the Lie subgroup of $K$ generated by $\mathfrak{u}$ is a normal subgroup isomorphic to $S p(1)$, its isotropy representation is isomorphic to the representation of the normal subgroup $S p(1)$ of $S p(n) S p(1)$ on $\mathbb{R}^{4 n}(4 n=\operatorname{dim} M)$, and there exists a normal subgroup $K_{1}$ of $K$ such that $K=K_{1} S p(1)$ and $K_{1} \cap S p(1)$ is contained in the center $\{ \pm 1\}$ of $S p(1)$. The Lie algebra $\mathfrak{k}_{1}$ of $K_{1}$ is an ideal of $\mathfrak{k}$ such that $\mathfrak{k}=\mathfrak{u} \oplus \mathfrak{k}_{1}$, and we can get a basis $\mathcal{B}=\left\{E_{1}, E_{2}, E_{3}, \ldots\right\}$ of $\mathfrak{k}$ with the basic elements of $\mathfrak{u}$ and some elements of $\mathfrak{k}_{1}$. Let $\left\{\alpha_{1}, \alpha_{2}, \alpha_{3}, \ldots\right\}$ be the dual basis of $\mathcal{B}$. Then we have 
Proposition 2.4. The homogeneous Riemannian structure $S$ on the connected non-compact quaternion-Kähler symmetric space $M=G / K$ associated to the reductive decomposition $\hat{\mathfrak{g}}=\mathfrak{h}+\mathfrak{m}$ satisfies equations (1.3) with 1-forms $\theta^{i}$, $i=1,2,3$, given at $o \equiv H \in \hat{G} / H \equiv M$ by

$$
\theta^{i}\left(X_{o}^{*}\right)=2 \alpha_{i}\left(\left(\xi\left(X_{\mathfrak{p}}\right)\right)_{\mathfrak{k}}\right),
$$

for each $X \in \mathfrak{a}+\mathfrak{n}$.

Proof. Let $X, Y \in \mathfrak{a}+\mathfrak{n}$. From the commutative diagram (2.5) we have $\left(J_{a}\right)_{o} Y_{o}^{*}$ $=\left(\operatorname{ad}_{E_{a}} Y_{\mathfrak{p}}\right)_{o}^{*}$. Then, from $(2.4)$, for each $a=1,2,3$, we get

$$
S_{X_{o}^{*}}\left(\left(J_{a}\right)_{o} Y_{o}^{*}\right)=\left[\left(\xi\left(X_{\mathfrak{p}}\right)\right)_{\mathfrak{k}}, \operatorname{ad}_{E_{a}} Y_{\mathfrak{p}}\right]_{o}^{*},
$$

and

$$
\left(J_{a}\right)_{o}\left(S_{X_{o}^{*}} Y_{o}^{*}\right)=\left(\operatorname{ad}_{E_{a}}\left[\left(\xi\left(X_{\mathfrak{p}}\right)\right)_{\mathfrak{k}}, Y_{\mathfrak{p}}\right]\right)_{o}^{*} .
$$

Hence, applying Jacobi's identity, we deduce

$$
S_{X_{o}^{*}}\left(\left(J_{a}\right)_{o} Y_{o}^{*}\right)-\left(J_{a}\right)_{o}\left(S_{X_{o}^{*}} Y_{o}^{*}\right)=-\left[\left[E_{a},\left(\xi\left(X_{\mathfrak{p}}\right)\right)_{\mathfrak{k}}\right], Y_{\mathfrak{p}}\right]_{o}^{*} .
$$

In terms of the basis $\mathcal{B}$, we have $\left(\xi\left(X_{\mathfrak{p}}\right)\right)_{\mathfrak{k}}=\alpha_{1}\left(\left(\xi\left(X_{\mathfrak{p}}\right)\right)_{\mathfrak{k}}\right) E_{1}+\alpha_{2}\left(\left(\xi\left(X_{\mathfrak{p}}\right)\right)_{\mathfrak{k}}\right) E_{2}+$ $\alpha_{3}\left(\left(\xi\left(X_{\mathfrak{p}}\right)\right)_{\mathfrak{k}}\right) E_{3}+\ldots$, then

$$
\left[E_{1},\left(\xi\left(X_{\mathfrak{p}}\right)\right)_{\mathfrak{k}}\right]=2 \alpha_{2}\left(\left(\xi\left(X_{\mathfrak{p}}\right)\right)_{\mathfrak{k}}\right) E_{3}-2 \alpha_{3}\left(\left(\xi\left(X_{\mathfrak{p}}\right)\right)_{\mathfrak{k}}\right) E_{2} .
$$

From (2.7), for instance for $a=1$, we have

$$
\begin{gathered}
S_{X_{o}^{*}}\left(\left(J_{1}\right)_{o} Y_{o}^{*}\right)-\left(J_{1}\right)_{o}\left(S_{X_{o}^{*}} Y_{o}^{*}\right)=-\left[2 \alpha_{2}\left(\left(\xi\left(X_{\mathfrak{p}}\right)\right)_{\mathfrak{k}}\right) E_{3}-2 \alpha_{3}\left(\left(\xi\left(X_{\mathfrak{p}}\right)\right)_{\mathfrak{k}}\right) E_{2}, Y_{\mathfrak{p}}\right]_{o}^{*} \\
\left.\left.=2 \alpha_{3}\left(\xi\left(X_{\mathfrak{p}}\right)\right)_{\mathfrak{k}}\right)\left[E_{2}, Y_{\mathfrak{p}}\right]_{o}^{*}-2 \alpha_{2}\left(\xi\left(X_{\mathfrak{p}}\right)\right)_{\mathfrak{k}}\right)\left[E_{3}, Y_{\mathfrak{p}}\right]_{o}^{*} \\
=2 \alpha_{3}\left(\left(\xi\left(X_{\mathfrak{p}}\right)\right)_{\mathfrak{k}}\right)\left(J_{2}\right)_{o} Y_{o}^{*}-2 \alpha_{2}\left(\left(\xi\left(X_{\mathfrak{p}}\right)\right)_{\mathfrak{k}}\right)\left(J_{3}\right)_{o} Y_{o}^{*}
\end{gathered}
$$

and equations (1.3) are satisfied, with $\theta^{i}\left(X_{o}^{*}\right), i=1,2,3$, as in the statement.

\section{$3 \quad$ Homogeneous descriptions and structures}

Since we want to obtain the types of homogeneous quaternionic Kähler structures on 8-dimensional non-compact quaternion-Kähler symmetric spaces, we need to get all the homogeneous descriptions of them.

As they are Alekseevsky spaces, we denote by $A_{S U(2,2)}, A_{S p(2,1)}$ and $A_{G_{2(2)}}$, the spaces $S U(2,2) / S(U(2) \times U(2)), \mathbb{H} H(2)=S p(2,1) /(S p(2) \times S p(1))$, and $G_{2(2)} / S O(4)$, respectively. (For $\mathbb{H} H(2)$ cf. [6, Ths. 1.1, 5.4]). Since the center of each of the corresponding full isometry groups is finite, Theorems 2.2 and 2.3 apply.

To find the corresponding homogeneous Riemannian structures, an important role will be played by the Lie algebra $\mathfrak{a}+\mathfrak{n}$ of the solvable factor $A N$ in an Iwasawa decomposition of the full connected isometry group of each space. 
To obtain each one of such structures $S$, we will use (2.4) and the identifications given by (2.2). In particular, we will also denote by $X \in \mathfrak{a}+\mathfrak{n}$ the vector $X_{o}^{*}=\left(X_{\mathfrak{p}}\right)_{o}^{*} \in T_{o}(M)$, and we will give the values $S_{X} Y=S_{X_{o}^{*}} Y_{o}^{*}$, for all $X, Y$ in a suitable basis of $\mathfrak{a}+\mathfrak{n}$. We know that $S$ is a homogeneous quaternionic Kähler structure and that Proposition 2.4 allows us to calculate directly the forms $\theta^{a}$ in (1.3).

\subsection{The complex hyperbolic space $A_{S U(2,2)}$}

\subsubsection{The quaternion-Hermitian structure of $\mathfrak{a}+\mathfrak{n} \subset \mathfrak{s u}(2,2)$}

The Lie algebra of $S U(2,2)$ is

$$
\mathfrak{s u}(2,2)=\left\{\left(\begin{array}{cc}
A & B \\
\bar{B}^{T} & C
\end{array}\right) \in \mathfrak{s l}(4, \mathbb{C}): A, C \in \mathfrak{u}(2)\right\} .
$$

The involution $\tau$ of $\mathfrak{s u}(2,2)$ given by $\tau(X)=-\bar{X}^{T}$ defines the Cartan decomposition $\mathfrak{s u}(2,2)=\mathfrak{k}+\mathfrak{p}$, where $\mathfrak{k}=\mathfrak{s}(\mathfrak{u}(2) \oplus \mathfrak{u}(2))$. We consider the subspace $\mathfrak{a}$ of $\mathfrak{p}$ defined by

$$
\mathfrak{a}=\left\{\left(\begin{array}{cccc}
0 & 0 & 0 & s_{2} \\
0 & 0 & s_{1} & 0 \\
0 & s_{1} & 0 & 0 \\
s_{2} & 0 & 0 & 0
\end{array}\right): s_{1}, s_{2} \in \mathbb{R}\right\},
$$

which is a maximal $\mathbb{R}$-diagonalizable subalgebra of $\mathfrak{s u}(2,2)$. Let $A_{1}$ and $A_{2}$ be the generators of $\mathfrak{a}$ defined by $\left(s_{1}, s_{2}\right)=(1,0)$ and $(0,1)$, respectively. Let $f_{1}$ and $f_{2}$ denote the linear functionals on $\mathfrak{a}$ whose values on the above matrix of $\mathfrak{a}$ are $s_{1}$ and $s_{2}$, respectively. Then, the sets of positive roots and simple roots (with respect to a suitable order in $\mathfrak{a}^{*}$ ) are given by $\Sigma^{+}=\left\{f_{1} \pm f_{2}, 2 f_{1}, 2 f_{2}\right\}$ and $\Pi=\left\{f_{1}-f_{2}, 2 f_{2}\right\}$, respectively. The positive root vector spaces are

$$
\begin{aligned}
\mathfrak{g}_{f_{1}+f_{2}}=\left\{\left(\begin{array}{cccc}
0 & z & -z & 0 \\
-\bar{z} & 0 & 0 & \bar{z} \\
-\bar{z} & 0 & 0 & \bar{z} \\
0 & z & -z & 0
\end{array}\right)\right\}, & \mathfrak{g}_{f_{1}-f_{2}}=\left\{\left(\begin{array}{ccccc}
0 & z & -z & 0 \\
-\bar{z} & 0 & 0 & -\bar{z} \\
-\bar{z} & 0 & 0 & -\bar{z} \\
0 & -z & z & 0
\end{array}\right)\right\}, \\
\mathfrak{g}_{2 f_{1}}=\left\{\left(\begin{array}{ccccc}
0 & 0 & 0 & 0 \\
0 & i x & -i x & 0 \\
0 & i x & -i x & 0 \\
0 & 0 & 0 & 0
\end{array}\right)\right\}, & \mathfrak{g}_{2 f_{2}}=\left\{\left(\begin{array}{cccc}
i x & 0 & 0 & -i x \\
0 & 0 & 0 & 0 \\
0 & 0 & 0 & 0 \\
i x & 0 & 0 & -i x
\end{array}\right)\right\},
\end{aligned}
$$

where $x \in \mathbb{R}, z \in \mathbb{C}$. The root vector spaces for the respective opposite roots are the corresponding sets of opposite conjugate transpose matrices. For each $f=f_{1} \pm f_{2}$, let $X_{f}$ and $X_{f}^{\prime}$ be the generators of $\mathfrak{g}_{f}$ in (3.1) obtained by putting $z=1$ and $z=i$, respectively, for each non-null entry. For $f=2 f_{j}$ $(1 \leq j \leq 2)$, let $U_{f}$ be the generator of $\mathfrak{g}_{f}$ in (3.2) obtained by putting $x=1$ in each non-zero entry. Let also $X_{f}, X_{f}^{\prime}, U_{f}$, be the corresponding elements of $\mathfrak{g}_{f}$ for the respective opposite roots $f \in \Sigma \backslash \Sigma^{+}$. Now, we put $X_{1}=X_{f_{1}+f_{2}}$, $Y_{1}=X_{-f_{1}-f_{2}}, X_{1}^{\prime}=X_{f_{1}+f_{2}}^{\prime}, Y_{1}^{\prime}=X_{-f_{1}-f_{2}}, X_{2}=X_{f_{1}-f_{2}}, Y_{2}=X_{-f_{1}+f_{2}}$, $X_{2}^{\prime}=X_{f_{1}-f_{2}}^{\prime}, Y_{2}^{\prime}=X_{-f_{1}+f_{2}}, U_{1}=U_{2 f_{1}}, V_{1}=U_{-2 f_{1}}, U_{2}=U_{2 f_{2}}, V_{2}=U_{-2 f_{2}}$. Moreover, the centralizer of $\mathfrak{a}$ in $\mathfrak{k}$ is generated by $C=\operatorname{diag}(i,-i,-i, i)$. Then, 
$Z_{\mathfrak{k}}(\mathfrak{a})+\mathfrak{a}=\left\langle C, A_{1}, A_{2}\right\rangle$ is a Cartan subalgebra of $\mathfrak{s u}(2,2)$, and $\mathfrak{s u}(2,2)=$ $\left(Z_{\mathfrak{k}}(\mathfrak{a})+\mathfrak{a}\right)+\sum_{f \in \Sigma} \mathfrak{g}_{f}$ is the restricted-root space decomposition. We also have the Iwasawa decomposition $\mathfrak{s u}(2,2)=\mathfrak{k}+\mathfrak{a}+\mathfrak{n}$, where $\mathfrak{n}=\sum_{f \in \Sigma^{+}} \mathfrak{g}_{f}=$ $\left\langle X_{1}, X_{1}^{\prime}, X_{2}, X_{2}^{\prime}, U_{1}, U_{2}\right\rangle$.

The elements $E_{1}, E_{2}, E_{3}$ of $\mathfrak{k}=\mathfrak{s}(\mathfrak{u}(2) \oplus \mathfrak{u}(2)) \subset \mathfrak{s u}(2,2)$ given by

$$
E_{1}=\left(\begin{array}{cccc}
0 & 0 & 0 & 0 \\
0 & 0 & 0 & 0 \\
0 & 0 & -i & 0 \\
0 & 0 & 0 & i
\end{array}\right), \quad E_{2}=\left(\begin{array}{cccc}
0 & 0 & 0 & 0 \\
0 & 0 & 0 & 0 \\
0 & 0 & 0 & -1 \\
0 & 0 & 1 & 0
\end{array}\right), \quad E_{3}=\left(\begin{array}{cccc}
0 & 0 & 0 & 0 \\
0 & 0 & 0 & 0 \\
0 & 0 & 0 & i \\
0 & 0 & i & 0
\end{array}\right),
$$

satisfy $\left[E_{1}, E_{2}\right]=2 E_{3},\left[E_{2}, E_{3}\right]=2 E_{1},\left[E_{3}, E_{1}\right]=2 E_{2}$. The compact subalgebra $\mathfrak{u} \cong \mathfrak{s p}(1)$ generated by $\left\{E_{1}, E_{2}, E_{3}\right\}$ is an ideal of $\mathfrak{k}$, and the isotropy representation $\mathfrak{u} \rightarrow \mathfrak{g l}(\mathfrak{p})$ defines a quaternionic structure on $A_{S U(2,2)}$. From the action of each $E_{i}$ on $\mathfrak{p}$ and through the isomorphisms $\mathfrak{p} \cong \mathfrak{s u}(2,2) / \mathfrak{k} \cong \mathfrak{a}+\mathfrak{n}$, we obtain the complex structures $J_{a}(a=1,2,3)$, acting on $\mathfrak{a}+\mathfrak{n}$. The action on the elements $A_{j}, U_{j}, X_{j}, X_{j}^{\prime}(j=1,2)$ of the basis of $\mathfrak{a}+\mathfrak{n}$ is the following.

\begin{tabular}{ccccccccc} 
& $A_{1}$ & $A_{2}$ & $U_{1}$ & $U_{2}$ & $X_{1}$ & $X_{1}^{\prime}$ & $X_{2}$ & $X_{2}^{\prime}$ \\
\hline$J_{1}$ & $-U_{1}$ & $U_{2}$ & $A_{1}$ & $-A_{2}$ & $X_{1}^{\prime}$ & $-X_{1}$ & $X_{2}^{\prime}$ & $-X_{2}$ \\
$J_{2}$ & $\frac{1}{2}\left(X_{1}-X_{2}\right)$ & $\frac{1}{2}\left(X_{1}+X_{2}\right)$ & $\frac{1}{2}\left(X_{1}^{\prime}-X_{2}^{\prime}\right)$ & $-\frac{1}{2}\left(X_{1}^{\prime}+X_{2}^{\prime}\right)$ & $-A_{1}-A_{2}$ & $-U_{1}+U_{2}$ & $A_{1}-A_{2}$ & $U_{1}+U_{2}$ \\
$J_{3}$ & $\frac{1}{2}\left(X_{1}^{\prime}-X_{2}^{\prime}\right)$ & $\frac{1}{2}\left(X_{1}^{\prime}+X_{2}^{\prime}\right)$ & $-\frac{1}{2}\left(X_{1}-X_{2}\right)$ & $\frac{1}{2}\left(X_{1}+X_{2}\right)$ & $U_{1}-U_{2}$ & $-A_{1}-A_{2}$ & $-U_{1}-U_{2}$ & $A_{1}-A_{2}$ \\
\hline
\end{tabular}

Any positive multiple of the restriction of the Killing form $B$ to $\mathfrak{p} \times \mathfrak{p}$ defines a Hermitian metric adapted to $\left(J_{1}, J_{2}, J_{3}\right)$. We consider the scalar product $\langle$,$\rangle in-$ duced in $\mathfrak{a}+\mathfrak{n}$ by the isomorphism $\mathfrak{p} \cong \mathfrak{a}+\mathfrak{n}$ and $\frac{1}{16} B_{\mid \mathfrak{p} \times \mathfrak{p}}$. This product makes the basis orthogonal, with $\left\langle A_{j}, A_{j}\right\rangle=\left\langle U_{j}, U_{j}\right\rangle=1,\left\langle X_{j}, X_{j}\right\rangle=\left\langle X_{j}^{\prime}, X_{j}^{\prime}\right\rangle=2$, and $\left(\mathfrak{a}+\mathfrak{n},\langle\rangle,, J_{1}, J_{2}, J_{3}\right)$ is a quaternion-Hermitian vector space.

\subsubsection{Homogeneous descriptions and structures of $A_{S U(2,2)}$}

We now determine the homogeneous descriptions and structures of $A_{S U(2,2)}$. These descriptions will be obtained from the parabolic subalgebras of $\mathfrak{s u}(2,2)$ and their refined Langland decompositions, by using Theorems 2.2 and 2.3. The four standard parabolic subalgebras are parametrized by the subsets $\Pi, \emptyset$, $\Psi_{1}=\left\{f_{1}-f_{2}\right\}$ and $\Psi_{2}=\left\{2 f_{2}\right\}$ of $\Pi$.

The case $\Psi=\Pi$. We have $\mathfrak{e}_{\Pi}^{\prime}=\mathfrak{a}_{\Pi}=\mathfrak{n}_{\Pi}=\{0\}$, hence the refined Langlands decomposition is $\mathfrak{p}_{\Pi}=\mathfrak{s u}(2,2)+\{0\}+\{0\}+\{0\}$. Thus, for a connected closed co-compact subgroup $\hat{G}$ of $P_{\Pi}$, it is only possible to have $\hat{G}=L=L_{\Pi}^{\prime}=$ $S U(2,2)$. Then the only transitive action on $A_{S U(2,2)}$ coming from $\Psi=\Pi$ is that of the full isometry group $S U(2,2)$, and we have the description of $A_{S U(2,2)}$ as the symmetric space $S U(2,2) / S(U(2) \times U(2))$. The associated reductive decomposition is the Cartan decomposition $\mathfrak{s u}(2,2)=\mathfrak{s}(\mathfrak{u}(2) \oplus \mathfrak{u}(2))+\mathfrak{p}$, and the corresponding homogeneous quaternionic Kähler structure is $S=0$.

The case $\Psi=\emptyset$. In this case, $\mathfrak{l}^{\prime}=\mathfrak{l}_{\emptyset}^{\prime}+\mathfrak{e}_{\emptyset}^{\prime}+\mathfrak{a}_{\emptyset}$ is commutative, $\mathfrak{l}_{\emptyset}^{\prime}=\{0\}$, $\mathfrak{e}_{\emptyset}^{\prime}=Z_{\mathfrak{k}}(\mathfrak{a})$, and $\mathfrak{a}_{\emptyset}=\mathfrak{a}$. Hence the refined Langlands decomposition of the minimal parabolic subalgebra is $\mathfrak{p}_{\emptyset}=\{0\}+Z_{\mathfrak{k}}(\mathfrak{a})+\mathfrak{a}+\mathfrak{n}$. For each connected 
closed subgroup $E$ of $E_{\emptyset}^{\prime} A \cong U(1) \times \mathbb{R}^{2}$ we get a co-compact subgroup $E N$ of $S U(2,2)$, where $N$ is the nilpotent factor in the Iwasawa decomposition of $S U(2,2)$. In order to obtain a transitive action it is sufficient that the projection of the Lie algebra $\mathfrak{e} \subset Z_{\mathfrak{k}}(\mathfrak{a})+\mathfrak{a}$ of $E$ to $\mathfrak{a}$ be surjective.

For each choice of a two-dimensional subspace $\mathfrak{e}$ of $Z_{\mathfrak{k}}(\mathfrak{a})+\mathfrak{a}=\left\langle C, A_{1}, A_{2}\right\rangle$ such as $C \notin \mathfrak{e}$, we obtain a Lie subalgebra $\mathfrak{e}+\mathfrak{n}$ of $\mathfrak{s u}(2,2)$ which generates a connected closed subgroup $\hat{G}=E N$ which acts simply transitively on $A_{S U(2,2)}$. We can suppose that $\mathfrak{e}=\mathfrak{e}_{\lambda, \mu}$ is generated by two elements of the form $\lambda C+A_{1}$, $\mu C+A_{2}$, with $\lambda, \mu \in \mathbb{R}$. This gives the reductive decomposition $\hat{\mathfrak{g}}^{\lambda, \mu}=\{0\}+\hat{\mathfrak{g}}^{\lambda, \mu}$ associated to the description $A_{S U(2,2)}=E_{\lambda, \mu} N$, where $\hat{\mathfrak{g}}^{\lambda, \mu}=\left\langle\lambda C+A_{1}, \mu C+\right.$ $\left.A_{2}, U_{1}, U_{2}, X_{1}, X_{1}^{\prime}, X_{2}, X_{2}^{\prime}\right\rangle$. Distinct values of $\lambda, \mu$ define non-isomorphic Lie algebras. If $\lambda=\mu=0$, we have the choice $\mathfrak{e}=\mathfrak{a}$, which gives the usual description of $A_{S U(2,2)}$ as the solvable Lie group $A N$.

We get a two-parameter family of structures $S^{\lambda, \mu}$ associated to the family of reductive decompositions $\hat{\mathfrak{g}}^{\lambda, \mu}=\{0\}+\hat{\mathfrak{g}}^{\lambda, \mu}$. With the identifications in (2.2), where now $\mathfrak{m}=\hat{\mathfrak{g}}^{\lambda, \mu}=\mathfrak{e}_{\lambda, \mu}+\mathfrak{n}$, and by using (2.4), we obtain that $S=S^{\lambda, \mu}$ is given at $o$ by Table I, with $\delta=\varepsilon=1$.

\begin{tabular}{ccccccccc} 
Table I & $A_{1}$ & $A_{2}$ & $U_{1}$ & $U_{2}$ & $X_{1}$ & $X_{1}^{\prime}$ & $X_{2}$ & $X_{2}^{\prime}$ \\
\hline$S_{A_{1}}$ & 0 & 0 & 0 & 0 & $2 \lambda X_{1}^{\prime}$ & $-2 \lambda X_{1}$ & $2 \lambda X_{2}^{\prime}$ & $-2 \lambda X_{2}$ \\
$S_{A_{2}}$ & 0 & 0 & 0 & 0 & $2 \mu X_{1}^{\prime}$ & $-2 \mu X_{1}$ & $2 \mu X_{2}^{\prime}$ & $-2 \mu X_{2}$ \\
$S_{U_{1}}$ & $-2 U_{1}$ & 0 & $2 A_{1}$ & 0 & $X_{2}^{\prime}$ & $-X_{2}$ & $X_{1}^{\prime}$ & $-X_{1}$ \\
$S_{U_{2}}$ & 0 & $-2 \delta U_{2}$ & 0 & $2 \delta A_{2}$ & $\delta X_{2}^{\prime}$ & $-\delta X_{2}$ & $\delta X_{1}^{\prime}$ & $-\delta X_{1}$ \\
$S_{X_{1}}$ & $-X_{1}$ & $-X_{1}$ & $X_{2}^{\prime}$ & $X_{2}^{\prime}$ & $2\left(A_{1}+A_{2}\right)$ & 0 & 0 & $-2\left(U_{1}+U_{2}\right)$ \\
$S_{X_{1}^{\prime}}$ & $-X_{1}^{\prime}$ & $-X_{1}^{\prime}$ & $-X_{2}$ & $-X_{2}$ & 0 & $2\left(A_{1}+A_{2}\right)$ & $2\left(U_{1}+U_{2}\right)$ & 0 \\
$S_{X_{2}}$ & $-\varepsilon X_{2}$ & $\varepsilon X_{2}$ & $\varepsilon X_{1}^{\prime}$ & $-\varepsilon X_{1}^{\prime}$ & 0 & $-2 \varepsilon\left(U_{1}-U_{2}\right)$ & $2 \varepsilon\left(A_{1}-A_{2}\right)$ & 0 \\
$S_{X_{2}^{\prime}}$ & $-\varepsilon X_{2}^{\prime}$ & $\varepsilon X_{2}^{\prime}$ & $-\varepsilon X_{1}$ & $\varepsilon X_{1}$ & $2 \varepsilon\left(U_{1}-U_{2}\right)$ & 0 & 0 & $2 \varepsilon\left(A_{1}-A_{2}\right)$ \\
\hline
\end{tabular}

Each structure $S^{\lambda, \mu}$ is also characterized by the fact that $\widetilde{\nabla}=\nabla-S^{\lambda, \mu}$ is the canonical connection for the Lie group $E_{\lambda, \mu} N$, which is the connection for which every left-invariant vector field on $E_{\lambda, \mu} N$ is parallel.

The choice $\mathfrak{e}=Z_{\mathfrak{k}}(\mathfrak{a})+\mathfrak{a}$ gives $A_{S U(2,2)} \equiv\left(U(1) \times \mathbb{R}^{2}\right) N / U(1)$, and the associated reductive decomposition is $\hat{\mathfrak{g}}=\mathfrak{h}+\mathfrak{m}$, where $\hat{\mathfrak{g}}=\mathfrak{p}_{\emptyset}, \mathfrak{h}=Z_{\mathfrak{k}}(\mathfrak{a})$ and $\mathfrak{m}=\mathfrak{a}+\mathfrak{n}$. The corresponding structure coincides with the above structure $S^{\lambda, \mu}$, for $\lambda=\mu=0$.

The case $\Psi=\Psi_{1}$. Then $\left[\Psi_{1}\right]=\left\{ \pm\left(f_{1}-f_{2}\right)\right\}$, and one has $\mathfrak{l}_{\Psi_{1}}^{\prime}=\left\langle A_{2}-\right.$ $\left.A_{1}\right\rangle+Z_{\mathfrak{k}}(\mathfrak{a})+\left\langle X_{2}, Y_{2}, X_{2}^{\prime}, Y_{2}^{\prime}\right\rangle, \mathfrak{e}^{\prime} \Psi_{1}=\{0\}, \mathfrak{a}_{\Psi_{1}}=\left\langle A_{1}+A_{2}\right\rangle$, and $\mathfrak{n}_{\Psi_{1}}=$ $\mathfrak{g}_{f_{1}+f_{2}}+\mathfrak{g}_{2 f_{1}}+\mathfrak{g}_{2 f_{2}}=\left\langle X_{1}, X_{1}^{\prime}, U_{1}, U_{2}\right\rangle$. Hence, the corresponding parabolic subalgebra is $\mathfrak{p}_{\Psi_{1}}=\mathfrak{l}_{\Psi_{1}}+\{0\}+\mathfrak{a}_{\Psi_{1}}+\mathfrak{n}_{\Psi_{1}}$, with

$$
\mathfrak{l}_{\Psi_{1}}^{\prime}=\left\{\left(\begin{array}{cccc}
i r & z & w & s \\
-\bar{z} & -i r & -s & \bar{w} \\
\bar{w} & -s & -i r & -\bar{z} \\
s & w & z & i r
\end{array}\right): r, s \in \mathbb{R}, z, w \in \mathbb{C}\right\} \cong \mathfrak{s l}(2, \mathbb{C}) .
$$

The connected subgroup $E=A_{\Psi_{1}} \cong \mathbb{R}$ of $P_{\Psi_{1}}$ with Lie algebra $\mathfrak{a}_{\Psi_{1}}$ is the only possible choice to get a co-compact subgroup $\hat{G}=L E N_{\Psi_{1}}$ of $S U(2,2)$, with $L=L_{\Psi_{1}}^{\prime} \cong S l(2, \mathbb{C})$, which acts transitively on $A_{S U(2,2)}$. The isotropy algebra is $\mathfrak{h}=\hat{\mathfrak{g}} \cap \mathfrak{k}=\mathfrak{l}_{\Psi_{1}}^{\prime} \cap \mathfrak{s}(\mathfrak{u}(2) \oplus \mathfrak{u}(2))=\left\langle C,\left(X_{2}\right)_{\mathfrak{k}},\left(X_{2}^{\prime}\right)_{\mathfrak{k}}\right\rangle \cong \mathfrak{s u}(2)$, and 
we get the reductive decomposition $\hat{\mathfrak{g}}=\mathfrak{h}+\mathfrak{m}$, where $\hat{\mathfrak{g}}=\mathfrak{p}_{\Psi_{1}}$, and $\mathfrak{m}=$ $\left\langle A_{1}, A_{2}, U_{1}, U_{2}, X_{1}, X_{1}^{\prime},\left(X_{2}\right)_{\mathfrak{p}},\left(X_{2}^{\prime}\right)_{\mathfrak{p}}\right\rangle$, which is associated to the description $A_{S U(2,2)} \equiv S l(2, \mathbb{C}) \mathbb{R} N_{\Psi_{1}} / S U(2)$. By using (2.4), we obtain that the corresponding structure $S$ is given at $o$ by Table I, with $\lambda=\mu=\varepsilon=0, \delta=1$.

The case $\Psi=\Psi_{2}$. Then $\left[\Psi_{2}\right]=\left\{ \pm 2 f_{2}\right\}$, and we have $\mathfrak{l}_{\Psi_{2}}^{\prime}=\left\langle A_{2}, U_{2}, V_{2}\right\rangle$, $\mathfrak{e}_{\Psi_{2}}^{\prime}=Z_{\mathfrak{k}}(\mathfrak{a}), \mathfrak{a}_{\Psi_{2}}=\left\langle A_{1}\right\rangle, \mathfrak{n}_{\Psi_{2}}=\mathfrak{g}_{f_{1}+f_{2}}+\mathfrak{g}_{f_{1}-f_{2}}+\mathfrak{g}_{2 f_{1}}=\left\langle X_{1}, X_{1}^{\prime}, X_{2}, X_{2}^{\prime}, U_{1}\right\rangle$. The corresponding parabolic subalgebra is $\mathfrak{p}_{\Psi_{2}}=\mathfrak{l}_{\Psi_{2}}^{\prime}+Z_{\mathfrak{k}}(\mathfrak{a})+\mathfrak{a}_{\Psi_{2}}+\mathfrak{n}_{\Psi_{2}}$, with

$$
\mathfrak{l}_{\Psi_{2}}^{\prime}=\left\{\left(\begin{array}{cccc}
i x & 0 & 0 & z \\
0 & 0 & 0 & 0 \\
0 & 0 & 0 & 0 \\
\bar{z} & 0 & 0 & -i x
\end{array}\right): x \in \mathbb{R}, z \in \mathbb{C}\right\} \cong \mathfrak{s u}(1,1) \cong \mathfrak{s l}(2, \mathbb{R}) .
$$

For each connected closed subgroup $E$ of $P_{\Psi_{2}}$ whose Lie algebra $\mathfrak{e}$ is a non-trivial subspace of $\mathfrak{e}_{\Psi_{2}}^{\prime}+\mathfrak{a}_{\Psi_{2}}$, $\mathfrak{e} \neq \mathfrak{e}_{\Psi_{2}}^{\prime}$, we get a co-compact subgroup $\hat{G}=L E N_{\Psi_{2}}$ of $S U(2,2)$, with $L=L_{\Psi_{2}}^{\prime} \cong S U(1,1) \cong S l(2, \mathbb{R})$, which acts transitively on $A_{S U(2,2)}$.

If $\operatorname{dim} E=1$, we get the description $A_{S U(2,2)} \equiv S U(1,1) \mathbb{R} N_{\Psi_{2}} / U(1)$. In fact, if $\mathfrak{e}$ is an one-dimensional subspace of $\mathfrak{e}_{\Psi_{2}}^{\prime}+\mathfrak{a}_{\Psi_{2}}$, with $\mathfrak{e} \neq \mathfrak{e}_{\Psi_{2}}^{\prime}$, then $\mathfrak{e}=$ $\mathfrak{e}_{\lambda}$ is spanned by an element of the form $\lambda C+A_{1}$, for $\lambda \in \mathbb{R}$, so one gets a one-parameter family of reductive decompositions $\hat{\mathfrak{g}}^{\lambda}=\mathfrak{h}+\mathfrak{m}^{\lambda}$, where $\mathfrak{h}=$ $\hat{\mathfrak{g}} \cap \mathfrak{k}=\mathfrak{l}_{\Psi_{2}}^{\prime} \cap \mathfrak{s}(\mathfrak{u}(2) \oplus \mathfrak{u}(2))=\left\langle\left(U_{2}\right)_{\mathfrak{k}}\right\rangle \cong \mathfrak{u}(1)$ is the isotropy algebra and $\mathfrak{m}^{\lambda}=\left\langle\lambda C+A_{1}, A_{2}, U_{1},\left(U_{2}\right)_{\mathfrak{p}}, X_{1}, X_{1}^{\prime}, X_{2}, X_{2}^{\prime}\right\rangle$. By (2.4), we have that the corresponding one-parameter family of structures $S^{\lambda}$ is given at $o$ by Table I, with $\mu=\delta=0, \varepsilon=1$. In particular, $\lambda=0$ corresponds to the choice $\mathfrak{e}=\mathfrak{a}_{\Psi_{2}}$.

If $\operatorname{dim} E=2$, then $E \cong U(1) \times \mathbb{R}$, which gives $A_{S U(2,2)} \equiv S U(1,1)(U(1) \times$ $\mathbb{R}) N_{\Psi_{2}} /(U(1) \times U(1))$. The natural associated reductive decomposition is $\hat{\mathfrak{g}}^{\prime}=$ $\mathfrak{h}^{\prime}+\mathfrak{m}^{\prime}$, where $\hat{\mathfrak{g}}=\mathfrak{p}_{\Psi_{2}}, \mathfrak{h}^{\prime}=\left\langle C,\left(U_{2}\right)_{\mathfrak{k}}\right\rangle \cong \mathfrak{u}(1) \oplus \mathfrak{u}(1), \mathfrak{m}^{\prime}=\left\langle A_{1}, A_{2}, U_{1},\left(U_{2}\right)_{\mathfrak{p}}\right.$, $\left.X_{1}, X_{1}^{\prime}, X_{2}, X_{2}^{\prime}\right\rangle$, and the corresponding structure $S^{\prime}$ coincides with the previous $S^{\lambda}$, for $\lambda=0$.

In particular, we have proved

Proposition 3.1. The space $A_{S U(2,2)}$ admits the homogeneous quaternionic Kähler structures $S$ (associated to the corresponding homogeneous description $\hat{G} / H)$ given by

\begin{tabular}{|l|l|}
\hline$S$ & $\hat{G} / H$ \\
\hline \hline 0 & $S U(2,2) / S(U(2) \times U(2))$ \\
Table I $(\lambda, \mu \in \mathbb{R} ; \delta=\varepsilon=1)$ & $E_{\lambda, \mu} N \quad\left(E_{\lambda, \mu} \cong \mathbb{R}^{2}\right)$ \\
Table I $(\lambda=\mu=\varepsilon=0, \delta=1)$ & $S l(2, \mathbb{C}) \mathbb{R} N_{\Psi_{1}} / S U(2)$ \\
Table I $(\lambda \in \mathbb{R} ; \mu=\delta=0, \varepsilon=1)$ & $S U(1,1) \mathbb{R} N_{\Psi_{2}} / U(1)$ \\
\hline
\end{tabular}

where $N$ is the nilpotent factor in the Iwasawa decomposition $S U(2,2)=S(U(2)$ $\times U(2)) A N$ and $\Psi_{1}$ and $\Psi_{2}$ are the subsets of simple restricted roots defining the intermediate parabolic subalgebras of $(\mathfrak{s u}(2,2), \mathfrak{a})$. 


\subsection{The quaternionic hyperbolic space $A_{S p(2,1)}=\mathbb{H} \mathrm{H}(2)$}

\subsubsection{The quaternion-Hermitian structure of $\mathfrak{a}+\mathfrak{n} \subset \mathfrak{s p}(2,1)$}

The Lie algebra $\mathfrak{s p}(2,1)$ can be described as the subalgebra of $\mathfrak{g l}(6, \mathbb{C})$ of all matrices $X \in \mathfrak{s p}(3, \mathbb{C})$ satisfying $\bar{X}^{T} J_{2,1}+J_{2,1} X=0$, where $J_{2,1}=\operatorname{diag}(-1,-1$, $1,-1,-1,1)$. The involution $\tau$ of $\mathfrak{s p}(2,1)$ given by $\tau(X)=-\bar{X}^{T}$ defines the Cartan decomposition $\mathfrak{s} \mathfrak{p}(2,1)=\mathfrak{k}+\mathfrak{p}$, where

$$
\begin{gathered}
\mathfrak{k}=\left\{\left(\begin{array}{rrrrrr}
i a & z & 0 & u & w & 0 \\
-\bar{z} & i b & 0 & w & v & 0 \\
0 & 0 & i c & 0 & 0 & \alpha \\
-\bar{u} & -\bar{w} & 0 & -i a & \bar{z} & 0 \\
-\bar{w} & -\bar{v} & 0 & -z & -i b & 0 \\
0 & 0 & -\bar{\alpha} & 0 & 0 & -i c
\end{array}\right): \begin{array}{r}
a, b, c \in \mathbb{R}, \\
z, u, v, w, \alpha \in \mathbb{C}
\end{array}\right\} \cong \mathfrak{s p}(2) \oplus \mathfrak{s p}(1), \\
\mathfrak{p}=\left\{\left(\begin{array}{rrrrrrr}
0 & 0 & p_{1} & 0 & 0 & q_{1} \\
0 & 0 & p_{2} & 0 & 0 & q_{2} \\
\bar{p}_{1} & \bar{p}_{2} & 0 & q_{1} & q_{2} & 0 \\
0 & 0 & \bar{q}_{1} & 0 & 0 & -\bar{p}_{1} \\
0 & 0 & \bar{q}_{2} & 0 & 0 & -\bar{p}_{2} \\
\bar{q}_{1} & \bar{q}_{2} & 0 & -p_{1} & -p_{2} & 0
\end{array}\right): p_{1}, p_{2}, q_{1}, q_{2} \in \mathbb{C}\right\}
\end{gathered}
$$

The element $A_{0}$ of $\mathfrak{p}$ obtained by taking $p_{1}=1$ and $p_{2}=q_{1}=q_{2}=0$ generates a maximal $\mathbb{R}$-diagonalizable subalgebra $\mathfrak{a}$ of $\mathfrak{s p}(2,1)$. The set of roots corresponding to $\mathfrak{a}$ is $\Sigma=\left\{ \pm f_{0}, \pm 2 f_{0}\right\}$, where $f_{0} \in \mathfrak{a}^{*}$ is given by $f_{0}\left(A_{0}\right)=1$. The set $\Pi=\left\{f_{0}\right\}$ is a system of simple roots and the corresponding positive root system is $\Sigma^{+}=\left\{f_{0}, 2 f_{0}\right\}$. The positive root vector spaces are

$$
\begin{aligned}
\mathfrak{g}_{f_{0}} & =\left\{\left(\begin{array}{rrrrrr}
0 & z & 0 & 0 & w & 0 \\
-\bar{z} & 0 & \bar{z} & w & 0 & w \\
0 & z & 0 & 0 & w & 0 \\
0 & -\bar{w} & 0 & 0 & \bar{z} & 0 \\
-\bar{w} & 0 & \bar{w} & -z & 0 & -z \\
0 & \bar{w} & 0 & 0 & -\bar{z} & 0
\end{array}\right): z, w \in \mathbb{C}\right\}, \\
\mathfrak{g}_{2 f_{0}} & =\left\{\left(\begin{array}{rrrrrr}
i a & 0 & -i a & u & 0 & u \\
0 & 0 & 0 & 0 & 0 & 0 \\
i a & 0 & -i a & u & 0 & u \\
-\bar{u} & 0 & \bar{u} & -i a & 0 & -i a \\
0 & 0 & 0 & 0 & 0 & 0 \\
\bar{u} & 0 & -\bar{u} & i a & 0 & i a
\end{array}\right): a \in \mathbb{R}, u \in \mathbb{C}\right\} .
\end{aligned}
$$

The root vector spaces for the respective opposite roots are the corresponding sets of opposite conjugate transpose matrices. Now, let $X_{1}, U_{1}$ and $U_{1}^{\prime}$ be the elements of $\mathfrak{g}_{2 f_{0}}$ in $(3.4)$ obtained by putting $(a, u)=(-1,0),(0,1)$ and $(0, i)$, respectively, and denote by $Y_{1}, V_{1}$ and $V_{1}^{\prime}$ the corresponding respective elements of $\mathfrak{g}_{-2 f_{0}}$. On the other hand, let $X_{2}, X_{2}^{\prime}, U_{2}$ and $U_{2}^{\prime}$ be the elements of $\mathfrak{g}_{f_{0}}$ in (3.3) obtained by setting $(z, w)=(1,0),(-i, 0),(0,1)$ and $(0, i)$, respectively, and let $Y_{2}, Y_{2}^{\prime}, V_{2}$ and $V_{2}^{\prime}$ denote the corresponding respective elements of $\mathfrak{g}_{-f_{0}}$. Then, $\mathfrak{g}_{f_{0}}=\left\langle X_{2}, X_{2}^{\prime}, U_{2}, U_{2}^{\prime}\right\rangle, \mathfrak{g}_{2 f_{0}}=\left\langle X_{1}, U_{1}, U_{1}^{\prime}\right\rangle, \mathfrak{g}_{-f_{0}}=\left\langle Y_{2}, Y_{2}^{\prime}, V_{2}, V_{2}^{\prime}\right\rangle, \mathfrak{g}_{-2 f_{0}}=$ $\left\langle Y_{1}, V_{1}, V_{1}^{\prime}\right\rangle$, and we have the Iwasawa decomposition $\mathfrak{s p}(2,1)=\mathfrak{k}+\mathfrak{a}+\mathfrak{n}$, where 
$\mathfrak{n}=\mathfrak{g}_{f_{0}}+\mathfrak{g}_{2 f_{0}}=\left\langle X_{1}, U_{1}, U_{1}^{\prime}, X_{2}, X_{2}^{\prime}, U_{2}, U_{2}^{\prime}\right\rangle$. The centralizer of $\mathfrak{a}$ in $\mathfrak{k}$ is

$$
Z_{\mathfrak{k}}(\mathfrak{a})=\left\{\left(\begin{array}{rrrrrr}
i a & 0 & 0 & u & 0 & 0 \\
0 & i b & 0 & 0 & v & 0 \\
0 & 0 & i a & 0 & 0 & -u \\
-\bar{u} & 0 & 0 & -i a & 0 & 0 \\
0 & -\bar{v} & 0 & 0 & -i b & 0 \\
0 & 0 & \bar{u} & 0 & 0 & -i a
\end{array}\right): \begin{array}{l}
a, b \in \mathbb{R}, \quad u, v \in \mathbb{C} \\
1
\end{array}\right\} \cong \mathfrak{s p}(1) \oplus \mathfrak{s p}(1),
$$

and it is generated by the elements $C_{1}, C_{2}, C_{3}, D_{1}, D_{2}$ and $D_{3}$ in (3.5) obtained by taking $(a, b, u, v)=(1,0,0,0),(0,0,1,0),(0,0, i, 0),(0,1,0,0),(0,0,0,1)$ and $(0,0,0, i)$, respectively. The subspaces $\left\langle C_{1}, C_{2}, C_{3}\right\rangle$ and $\left\langle D_{1}, D_{2}, D_{3}\right\rangle$ are ideals of $Z_{\mathfrak{k}}(\mathfrak{a})$ isomorphic to $\mathfrak{s p}(1)$.

The elements $E_{1}, E_{2}, E_{3}$ of $\mathfrak{k}=\mathfrak{s p}(2) \oplus \mathfrak{s p}(1) \subset \mathfrak{s p}(2,1)$ given by

$$
\left(\begin{array}{cccccc}
0 & 0 & 0 & 0 & 0 & 0 \\
0 & 0 & 0 & 0 & 0 & 0 \\
0 & 0 & i & 0 & 0 & 0 \\
0 & 0 & 0 & 0 & 0 & 0 \\
0 & 0 & 0 & 0 & 0 & 0 \\
0 & 0 & 0 & 0 & 0 & 0 \\
0 & 0 & 0 & 0 & 0 & -i
\end{array}\right), \quad\left(\begin{array}{cccccc}
0 & 0 & 0 & 0 & 0 & 0 \\
0 & 0 & 0 & 0 & 0 & 0 \\
0 & 0 & 0 & 0 & 0 & 1 \\
0 & 0 & 0 & 0 & 0 & 0 \\
0 & 0 & 0 & 0 & 0 & 0 \\
0 & 0 & 0 & 0 & 0 & 0 \\
0 & 0 & -1 & 0 & 0 & 0
\end{array}\right), \quad\left(\begin{array}{cccccc}
0 & 0 & 0 & 0 & 0 & 0 \\
0 & 0 & 0 & 0 & 0 & 0 \\
0 & 0 & 0 & 0 & 0 & i \\
0 & 0 & 0 & 0 & 0 & 0 \\
0 & 0 & 0 & 0 & 0 & 0 \\
0 & 0 & 0 & 0 & 0 & 0 \\
0 & 0 & i & 0 & 0 & 0
\end{array}\right),
$$

respectively, satisfy $\left[E_{1}, E_{2}\right]=2 E_{3},\left[E_{2}, E_{3}\right]=2 E_{1},\left[E_{3}, E_{1}\right]=2 E_{2}$, and generate a compact ideal $\mathfrak{u} \cong \mathfrak{s p}(1)$ of $\mathfrak{k}$. The isotropy representation $\mathfrak{u} \rightarrow \mathfrak{g l}(\mathfrak{p})$ defines a quaternionic structure on $A_{S p(2,1)}$. The isomorphisms $\mathfrak{p} \cong \mathfrak{s p}(2,1) / \mathfrak{k} \cong$ $\mathfrak{a}+\mathfrak{n}$ allow to obtain the complex structures $J_{a}(a=1,2,3)$, acting on $\mathfrak{a}+\mathfrak{n}$. The action on the elements of the basis $\left\{A_{0}, X_{1}, U_{1}, U_{1}^{\prime}, X_{2}, X_{2}^{\prime}, U_{2}, U_{2}^{\prime}\right\}$ of $\mathfrak{a}+\mathfrak{n}$ is given in the following table.

\begin{tabular}{ccccccccc} 
& $A_{0}$ & $X_{1}$ & $U_{1}$ & $U_{1}^{\prime}$ & $X_{2}$ & $X_{2}^{\prime}$ & $U_{2}$ & $U_{2}^{\prime}$ \\
\hline$J_{1}$ & $-X_{1}$ & $A_{0}$ & $U_{1}^{\prime}$ & $-U_{1}$ & $-X_{2}^{\prime}$ & $X_{2}$ & $U_{2}^{\prime}$ & $-U_{2}$ \\
$J_{2}$ & $-U_{1}$ & $-U_{1}^{\prime}$ & $A_{0}$ & $X_{1}$ & $-U_{2}$ & $-U_{2}^{\prime}$ & $X_{2}$ & $X_{2}^{\prime}$ \\
$J_{3}$ & $-U_{1}^{\prime}$ & $U_{1}$ & $-X_{1}$ & $A_{0}$ & $-U_{2}^{\prime}$ & $U_{2}$ & $-X_{2}^{\prime}$ & $X_{2}$ \\
\hline
\end{tabular}

The above basis of $\mathfrak{a}+\mathfrak{n}$ is orthonormal with respect to the scalar product $\langle$,$\rangle induced in \mathfrak{a}+\mathfrak{n}$ by the isomorphism $\mathfrak{p} \cong \mathfrak{a}+\mathfrak{n}$ and $\frac{1}{32} B_{\mid \mathfrak{p} \times \mathfrak{p}}$, where $B$ is the Killing form of $\mathfrak{s p}(2,1)$, and $\left(\mathfrak{a}+\mathfrak{n},\langle\rangle,, J_{1}, J_{2}, J_{3}\right)$ is a quaternion-Hermitian vector space.

\subsubsection{Homogeneous descriptions and structures of $A_{S p(2,1)}$}

We now determine the homogeneous descriptions and structures of $A_{S p(2,1)}$. The two only parabolic subalgebras of $\mathfrak{s p}(2,1)$ are parametrized by the subsets $\Pi$ and $\emptyset$ of $\Pi=\left\{f_{0}\right\}$. We will use them and their refined Langland decompositions to obtain, by means of Theorems 2.2 and 2.3, the homogeneous descriptions of $A_{S p(2,1)}=\mathbb{H} \mathrm{H}(2)$.

The case $\Psi=\Pi$. We have $\mathfrak{e}_{\Pi}^{\prime}=\mathfrak{a}_{\Pi}=\mathfrak{n}_{\Pi}=\{0\}$, hence the refined Langlands decomposition is $\mathfrak{p}_{\Pi}=\mathfrak{s p}(2,1)+\{0\}+\{0\}+\{0\}$. Theorem 2.2 then says that the only connected closed co-compact subgroup of $P_{\Pi}$ is $\hat{G}=S p(2,1)$. Thus the only transitive action coming from $\Psi=\Pi$ is that of the full isometry group $S p(2,1)$. This gives the description of $A_{S p(2,1)}=\mathbb{H H}(2)$ as the symmetric space 
$S p(2,1) /(S p(2) \times S p(1))$. The associated reductive decomposition is the Cartan decomposition $\mathfrak{s p}(2,1)=(\mathfrak{s p}(2) \oplus \mathfrak{s p}(1))+\mathfrak{p}$, and the corresponding structure is $S=0$.

The case $\Psi=\emptyset$. We have $\mathfrak{l}^{\prime}=\mathfrak{a}+Z_{\mathfrak{k}}(\mathfrak{a})=\mathfrak{l}_{\emptyset}^{\prime}+\mathfrak{e}_{\emptyset}^{\prime}+\mathfrak{a}_{\emptyset}$, with $\mathfrak{l}_{\emptyset}^{\prime}=\{0\}$, $\mathfrak{e}_{\emptyset}^{\prime}=Z_{\mathfrak{k}}(\mathfrak{a}), \mathfrak{a}_{\emptyset}=\mathfrak{a}$, so the refined Langlands decomposition of the corresponding parabolic subalgebra is $\mathfrak{p}_{\emptyset}=\{0\}+Z_{\mathfrak{k}}(\mathfrak{a})+\mathfrak{a}+\mathfrak{n}=\{0\}+(\mathfrak{s p}(1) \oplus \mathfrak{s p}(1))+$ $\mathfrak{a}+\left(\mathfrak{g}_{f_{0}}+\mathfrak{g}_{2 f_{0}}\right)$. For each connected closed subgroup $E$ of $E_{\emptyset}^{\prime} A \cong S p(1) S p(1) \mathbb{R}$ we get a co-compact subgroup $E N$ of $S p(2,1)$. In order to get a transitive action on $A_{S p(2,1)}$ it is sufficient, by Theorem 2.3, that the projection of the Lie algebra $\mathfrak{e} \subset Z_{\mathfrak{k}}(\mathfrak{a})+\mathfrak{a}$ to $\mathfrak{a}$ be surjective.

Now, for each one-dimensional subspace $\mathfrak{e}$ of $Z_{\mathfrak{k}}(\mathfrak{a})+\mathfrak{a}=\left\langle C_{j}, D_{j}, A_{0}\right\rangle_{j=1,2,3}$ such that the projection of $\mathfrak{e}$ to $\mathfrak{a}$ is an isomorphism, we get a Lie subalgebra $\mathfrak{e}+\mathfrak{n}$ of $\mathfrak{s p}(2,1)$ which generates a connected closed Lie subgroup $\hat{G}=E N$ acting simply transitively on $A_{S p(2,1)}$. We can suppose that $\mathfrak{e}=\mathfrak{e}_{\lambda, \mu}$ is generated by one element of the form $\hat{A}_{0}=A_{0}+\sum_{j=1}^{3}\left(\lambda_{j} C_{j}+\mu_{j} D_{j}\right)$, with $\lambda=\left(\lambda_{1}, \lambda_{2}, \lambda_{3}\right), \mu=\left(\mu_{1}, \mu_{2}, \mu_{3}\right) \in \mathbb{R}^{3}$. This gives the reductive decomposition $\hat{\mathfrak{g}}^{\lambda, \mu}=\{0\}+\hat{\mathfrak{g}}^{\lambda, \mu}$ associated to the description $A_{S p(2,1)}=E_{\lambda, \mu} N$, where $\hat{\mathfrak{g}}^{\lambda, \mu}=\left\langle\hat{A}_{0}, X_{1}, U_{1}, U_{1}^{\prime}, X_{2}, X_{2}^{\prime}, U_{2}, U_{2}^{\prime}\right\rangle$. If $\lambda=\mu=(0,0,0)$, we have $\mathfrak{e}=\mathfrak{a}$, which gives the usual description of $A_{S p(2,1)}$ as the solvable Lie group $A N$. Then we have a six-parameter family of structures $S=S^{\lambda, \mu}$ associated to the reductive decompositions $\hat{\mathfrak{g}}^{\lambda, \mu}=\{0\}+\hat{\mathfrak{g}}^{\lambda, \mu}$. For each $\lambda, \mu \in \mathbb{R}^{3}$, and with the identifications in (2.2), where now $\mathfrak{m}=\hat{\mathfrak{g}}^{\lambda, \mu}=\mathfrak{e}_{\lambda, \mu}+\mathfrak{n}$, we apply (2.4) to obtain the homogeneous quaternionic Kähler structure $S=S^{\lambda, \mu}$, which is given at $o$ by Table II.

\begin{tabular}{|c|c|c|c|c|c|c|c|c|}
\hline Table II & $A_{0}$ & $x_{1}$ & $U_{1}$ & $U_{1}^{\prime}$ & $x_{2}$ & $x_{2}^{\prime}$ & $U_{2}$ & $U_{2}^{\prime}$ \\
\hline$S_{A_{0}}$ & 0 & $\begin{array}{c}2 \lambda_{2} U_{1}^{\prime} \\
-2 \lambda_{3} U_{1}\end{array}$ & $\begin{array}{c}2 \lambda_{1} U_{1}^{\prime} \\
+2 \lambda_{3} X_{1}\end{array}$ & $\begin{array}{l}-2 \lambda_{1} U_{1} \\
-2 \lambda_{2} X_{1}\end{array}$ & $\begin{aligned} & \left(\mu_{1}-\lambda_{1}\right) X_{2}^{\prime} \\
+ & \left(\lambda_{2}-\mu_{2}\right) U_{2} \\
& +\left(\lambda_{3}-\mu_{3}\right) U_{2}^{\prime}\end{aligned}$ & $\begin{aligned} & \left(\lambda_{1}-\mu_{1}\right) X_{2} \\
+ & \left(\lambda_{2}+\mu_{2}\right) U_{2}^{\prime} \\
- & \left(\lambda_{3}+\mu_{3}\right) U_{2}\end{aligned}$ & $\begin{aligned} & \left(\lambda_{1}+\mu_{1}\right) U_{2}^{\prime} \\
+ & \left(\mu_{2}-\lambda_{2}\right) X_{2} \\
+ & \left(\lambda_{3}+\mu_{3}\right) X_{2}^{\prime}\end{aligned}$ & $\begin{array}{l}-\left(\lambda_{1}+\mu_{1}\right) U_{2} \\
-\left(\lambda_{2}+\mu_{2}\right) X_{2}^{\prime} \\
+\left(\mu_{3}-\lambda_{3}\right) X_{2}\end{array}$ \\
\hline$s_{X_{1}}$ & $-2 X_{1}$ & $2 A_{0}$ & 0 & 0 & $-X_{2}^{\prime}$ & $X_{2}$ & $U_{2}^{\prime}$ & $-U_{2}$ \\
\hline$S_{U_{1}}^{\Lambda_{1}}$ & $-2 U_{1}$ & 0 & $2 A_{0}$ & 0 & $-U_{2}^{2}$ & $-U_{2}^{\prime}$ & $x_{2}$ & $X_{2}^{\prime}$ \\
\hline$S_{U_{1}^{\prime}}$ & $-2 U_{1}^{\prime}$ & 0 & 0 & $2 A_{0}$ & $-U_{2}^{\prime}$ & $U_{2}$ & $-X_{2}^{\prime}$ & $x_{2}$ \\
\hline$s_{X_{2}}$ & $-X_{2}$ & $-X_{2}^{\prime}$ & $-U_{2}$ & $-U_{2}^{\prime}$ & $A_{0}$ & $x_{1}$ & $U_{1}$ & $U_{1}^{\prime}$ \\
\hline$s_{X_{2}^{\prime}}$ & $-X_{2}^{\prime}$ & $x_{2}$ & $-U_{2}^{\prime}$ & $U_{2}$ & $-X_{1}$ & $A_{0}$ & $-U_{1}^{\prime}$ & $U_{1}$ \\
\hline $\begin{array}{l}\mathrm{A}_{2} \\
S_{U_{2}}\end{array}$ & $-U_{2}$ & $U_{2}^{\prime}$ & $x_{2}$ & $-X_{2}^{\prime}$ & $-U_{1}$ & $U_{1}^{\prime}$ & $A_{0}$ & $-X_{1}$ \\
\hline$S_{U_{2}^{\prime}}$ & $-U_{2}^{\prime}$ & $-U_{2}$ & $X_{2}^{\prime}$ & $x_{2}$ & $-U_{1}^{\prime}$ & $\begin{array}{l}1 \\
-U_{1}\end{array}$ & $x_{1}$ & $A_{0}$ \\
\hline
\end{tabular}

If $\operatorname{dim} \mathfrak{e}>1$ we obtain other subgroups $E$ of $E_{\emptyset}^{\prime} A \cong S p(1) S p(1) \mathbb{R}$ such that $E N$ acts transitively on $A_{S p(2,1)}$. Such a group $E$ is isomorphic to some group of the form $U(1) \mathbb{R}, U(1) U(1) \mathbb{R}, S p(1) \mathbb{R}, S p(1) U(1) \mathbb{R}$ or $S p(1) S p(1) \mathbb{R}$. However, the natural reductive decompositions defined by their actions do not provide new homogeneous Riemannian structures.

We have proved

Proposition 3.2. The space $A_{S p(2,1)}$ admits the homogeneous quaternionic Kähler structures $S$ (associated to each corresponding homogeneous description 
$\hat{G} / H)$ given by

\begin{tabular}{|c|c|c|}
\hline $\bar{S}$ & & $\hat{G} / H$ \\
\hline $\begin{array}{l}0 \\
\text { Table II }\end{array}$ & $\left(\lambda, \mu \in \mathbb{R}^{3}\right)$ & $\begin{array}{l}S p(2,1) /(S p(2) \times S p(1)) \\
E_{\lambda, \mu} N \quad\left(E_{\lambda, \mu} \cong \mathbb{R}\right) \\
\end{array}$ \\
\hline
\end{tabular}

where $N$ is the nilpotent factor in the Iwasawa decomposition of $\operatorname{Sp}(2,1)$.

\subsection{The exceptional space $A_{G_{2(2)}}=G_{2(2)} / S O(4)$}

\subsubsection{The quaternion-Hermitian structure of $\mathfrak{a}+\mathfrak{n} \subset \mathfrak{g}_{2(2)}$}

The non-compact real form $\mathfrak{g}_{2(2)}$ of the exceptional Lie algebra $\mathfrak{g}_{2}$ can be realized as a subalgebra of $\mathfrak{s o}(4,3)$. Each element $X \in \mathfrak{g}_{2(2)}$ can be represented by a real matrix of the form

$$
\left(\begin{array}{ccccccc}
0 & x_{1}+u_{1} & -x_{2}-u_{2} & 2 u_{3} & s_{1} & y_{1}+v_{1} & y_{2}-v_{2} \\
-x_{1}-u_{1} & 0 & x_{3}+u_{3} & 2 u_{2} & y_{1}-v_{1} & s_{2} & y_{3}+v_{3} \\
x_{2}+u_{2} & -x_{3}-u_{3} & 0 & 2 u_{1} & y_{2}+v_{2} & y_{3}-v_{3} & s_{3} \\
-2 u_{3} & -2 u_{2} & -2 u_{1} & 0 & 2 v_{3} & 2 v_{2} & 2 v_{1} \\
s_{1} & y_{1}-v_{1} & y_{2}+v_{2} & 2 v_{3} & 0 & x_{1}-u_{1} & -x_{2}+u_{2} \\
y_{1}+v_{1} & s_{2} & y_{3}-v_{3} & 2 v_{2} & -x_{1}+u_{1} & 0 & x_{3}-u_{3} \\
y_{2}-v_{2} & y_{3}+v_{3} & s_{3} & 2 v_{1} & x_{2}-u_{2} & -x_{3}+u_{3} & 0
\end{array}\right)
$$

with $s_{1}+s_{2}+s_{3}=0$. The involution $\tau$ of $\mathfrak{g}_{2(2)}$ given by $\tau(X)=-X^{T}$ defines the Cartan decomposition $\mathfrak{g}_{2(2)}=\mathfrak{k}+\mathfrak{p}$, where $\mathfrak{k}=(\mathfrak{s o}(4) \oplus \mathfrak{s o}(3)) \cap \mathfrak{g}_{2(2)} \cong \mathfrak{s o}(4)$. The subspace $\mathfrak{a}$ of $\mathfrak{p}$ defined by

$$
\mathfrak{a}=\left\{\left(\begin{array}{ccccccc}
0 & 0 & 0 & 0 & s_{1} & 0 & 0 \\
0 & 0 & 0 & 0 & 0 & s_{2} & 0 \\
0 & 0 & 0 & 0 & 0 & 0 & s_{3} \\
0 & 0 & 0 & 0 & 0 & 0 & 0 \\
s_{1} & 0 & 0 & 0 & 0 & 0 & 0 \\
0 & s_{2} & 0 & 0 & 0 & 0 & 0 \\
0 & 0 & s_{3} & 0 & 0 & 0 & 0
\end{array}\right): s_{1}+s_{2}+s_{3}=0\right\}
$$

is a maximal $\mathbb{R}$-diagonalizable subalgebra of $\mathfrak{g}_{2(2)}$, and $Z_{\mathfrak{k}}(\mathfrak{a})=\{0\}$. The elements $A_{1}$ and $A_{2}$ of $\mathfrak{a}$ defined by $\left(s_{1}, s_{2}, s_{3}\right)=(1,-1,0)$ and $(-2,1,1)$, respectively, generate $\mathfrak{a}$. Let $f_{j}$ be the member of $\mathfrak{a}^{*}$ whose value on the above matrix of $\mathfrak{a}$ is $s_{j}$. Then $f_{1}+f_{2}+f_{3}=0$. We consider the (weak) order in $\mathfrak{a}^{*}$ defined by choosing the element $A_{0} \in \mathfrak{a}$ given by $\left(s_{1}, s_{2}, s_{3}\right)=(-1,-2,3)$ and by saying that $f \in \mathfrak{a}^{*}$ is positive if $f\left(A_{0}\right)>0$. Then, the sets of positive roots and simple roots of $\left(\mathfrak{g}_{2(2)}, \mathfrak{a}\right)$ are $\Sigma^{+}=\left\{f_{1}-f_{2}, f_{3}-f_{1}, f_{3}-f_{2}, f_{3},-f_{2},-f_{1}\right\}$ and $\Pi=\left\{f_{1}-f_{2},-f_{1}\right\}$, respectively. Now, we will give the generators $X_{j}, Y_{j}, U_{j}, V_{j}, 1 \leq j \leq 3$, of the root spaces $\mathfrak{g}_{f}, f \in \Sigma$. The matrix $X$ in (3.6) represents

$$
\begin{aligned}
& X_{1} \text { if } x_{1}=y_{1}=\frac{1}{2}, \quad X_{2} \text { if } x_{2}=y_{2}=\frac{1}{2}, \quad X_{3} \text { if } x_{3}=-y_{3}=-\frac{1}{2}, \\
& Y_{1} \text { if } x_{1}=-y_{1}=-\frac{1}{2}, \quad Y_{2} \text { if } x_{2}=-y_{2}=-\frac{1}{2}, \quad Y_{3} \text { if } x_{3}=y_{3}=-\frac{1}{2} \text {, } \\
& U_{1} \text { if } u_{1}=v_{1}=-\frac{1}{2}, \quad U_{2} \text { if } u_{2}=-v_{2}=\frac{1}{2}, \quad U_{3} \text { if } u_{3}=-v_{3}=-\frac{1}{2} \text {, } \\
& V_{1} \text { if } u_{1}=-v_{1}=\frac{1}{2}, \quad V_{2} \text { if } u_{2}=v_{2}=-\frac{1}{2}, \quad V_{3} \text { if } u_{3}=v_{3}=\frac{1}{2} \text {, }
\end{aligned}
$$


with all the other values $x_{j}, y_{j}, u_{j}, v_{j}, s_{j}=0$ for each of the 12 cases. We have $\mathfrak{g}_{f_{1}-f_{2}}=\left\langle X_{1}\right\rangle, \mathfrak{g}_{-f_{1}+f_{2}}=\left\langle Y_{1}\right\rangle, \mathfrak{g}_{f_{3}-f_{1}}=\left\langle X_{2}\right\rangle, \mathfrak{g}_{-f_{3}+f_{1}}=\left\langle Y_{2}\right\rangle$, $\mathfrak{g}_{f_{3}-f_{2}}=\left\langle X_{3}\right\rangle, \mathfrak{g}_{-f_{3}+f_{2}}=\left\langle Y_{3}\right\rangle, \mathfrak{g}_{f_{3}}=\left\langle U_{1}\right\rangle, \mathfrak{g}_{-f_{3}}=\left\langle V_{1}\right\rangle, \mathfrak{g}_{-f_{2}}=\left\langle U_{2}\right\rangle$, $\mathfrak{g}_{f_{2}}=\left\langle V_{2}\right\rangle, \mathfrak{g}_{-f_{1}}=\left\langle U_{3}\right\rangle, \mathfrak{g}_{f_{1}}=\left\langle V_{3}\right\rangle$. Then $\mathfrak{g}_{2(2)}=\mathfrak{a}+\sum_{f \in \Sigma} \mathfrak{g}_{f}$, and we have the Iwasawa decomposition $\mathfrak{g}_{2(2)}=\mathfrak{k}+\mathfrak{a}+\mathfrak{n}$, where $\mathfrak{n}=\sum_{f \in \Sigma^{+}} \mathfrak{g}_{f}=$ $\left\langle X_{1}, X_{2}, X_{3}, U_{1}, U_{2}, U_{3}\right\rangle$.

The elements $E_{1}, E_{2}, E_{3}$ of $\mathfrak{k}=\mathfrak{s o}(4) \subset \mathfrak{g}_{2(2)}$, which are represented by the matrices

$$
\left(\begin{array}{ccccccc}
0 & 1 & 0 & 0 & 0 & 0 & 0 \\
-1 & 0 & 0 & 0 & 0 & 0 & 0 \\
0 & 0 & 0 & 1 & 0 & 0 & 0 \\
0 & 0 & -1 & 0 & 0 & 0 & 0 \\
0 & 0 & 0 & 0 & 0 & 0 & 0 \\
0 & 0 & 0 & 0 & 0 & 0 & 0 \\
0 & 0 & 0 & 0 & 0 & 0 & 0
\end{array}\right),\left(\begin{array}{ccccccc}
0 & 0 & -1 & 0 & 0 & 0 & 0 \\
0 & 0 & 0 & 1 & 0 & 0 & 0 \\
1 & 0 & 0 & 0 & 0 & 0 & 0 \\
0 & -1 & 0 & 0 & 0 & 0 & 0 \\
0 & 0 & 0 & 0 & 0 & 0 & 0 \\
0 & 0 & 0 & 0 & 0 & 0 & 0 \\
0 & 0 & 0 & 0 & 0 & 0 & 0
\end{array}\right),\left(\begin{array}{ccccccc}
0 & 0 & 0 & 1 & 0 & 0 & 0 \\
0 & 0 & 1 & 0 & 0 & 0 & 0 \\
0 & -1 & 0 & 0 & 0 & 0 & 0 \\
-1 & 0 & 0 & 0 & 0 & 0 & 0 \\
0 & 0 & 0 & 0 & 0 & 0 & 0 \\
0 & 0 & 0 & 0 & 0 & 0 & 0 \\
0 & 0 & 0 & 0 & 0 & 0 & 0
\end{array}\right),
$$

respectively, satisfy $\left[E_{1}, E_{2}\right]=2 E_{3},\left[E_{2}, E_{3}\right]=2 E_{1},\left[E_{3}, E_{1}\right]=2 E_{2}$, and generate a compact ideal $\mathfrak{u} \cong \mathfrak{s p}(1)$ of $\mathfrak{k}$, such that the isotropy representation $\mathfrak{u} \rightarrow \mathfrak{g l}(\mathfrak{p})$ defines a quaternionic structure on $A_{G_{2(2)}}$. Through the isomorphisms $\mathfrak{p} \cong \mathfrak{g}_{2(2)} / \mathfrak{k} \cong \mathfrak{a}+\mathfrak{n}$, the action of each $E_{i}$ on $\mathfrak{p}$ defines the complex structure $J_{a}(a=1,2,3)$, acting on the elements $A_{1}, A_{2}, X_{j}, U_{j}(1 \leq j \leq 3)$ of $\mathfrak{a}+\mathfrak{n}$ as follows.

\begin{tabular}{ccccccccc} 
& $A_{1}$ & $A_{2}$ & $X_{1}$ & $X_{2}$ & $X_{3}$ & $U_{1}$ & $U_{2}$ & $U_{3}$ \\
\hline$J_{1}$ & $-2 X_{1}$ & $3 X_{1}+U_{1}$ & $\frac{1}{2} A_{1}$ & $\frac{1}{2}\left(X_{3}-U_{3}\right)$ & $-\frac{1}{2}\left(X_{2}+U_{2}\right)$ & $-\frac{3}{2} A_{1}-A_{2}$ & $\frac{1}{2}\left(3 X_{3}+U_{3}\right)$ & $\frac{1}{2}\left(3 X_{2}-U_{2}\right)$ \\
$J_{2}$ & $X_{2}-U_{2}$ & $-3 X_{2}+U_{2}$ & $-\frac{1}{2}\left(X_{3}+U_{3}\right)$ & $\frac{1}{2}\left(A_{1}+A_{2}\right)$ & $\frac{1}{2}\left(X_{1}-U_{1}\right)$ & $\frac{1}{2}\left(3 X_{3}-U_{3}\right)$ & $\frac{1}{2}\left(3 A_{1}+A_{2}\right)$ & $\frac{1}{2}\left(3 X_{1}+U_{1}\right)$ \\
$J_{3}$ & $-X_{3}-U_{3}$ & $2 U_{3}$ & $\frac{1}{2}\left(-X_{2}+U_{2}\right)$ & $\frac{1}{2}\left(X_{1}+U_{1}\right)$ & $A_{1}+\frac{1}{2} A_{2}$ & $-\frac{1}{2}\left(3 X_{2}+U_{2}\right)$ & $\frac{1}{2}\left(-3 X_{1}+U_{1}\right)$ & $-\frac{1}{2} A_{2}$ \\
\hline
\end{tabular}

We consider the scalar product $\langle$,$\rangle induced in \mathfrak{a}+\mathfrak{n}$ by the isomorphisms $\mathfrak{p} \cong \mathfrak{a}+\mathfrak{n}$ and $\frac{1}{16} B_{\mid \mathfrak{p} \times \mathfrak{p}}$, where $B$ is the Killing form of $\mathfrak{g}_{2(2)}$. Then, $\left\langle A_{1}, A_{1}\right\rangle=$ $1,\left\langle A_{2}, A_{2}\right\rangle=3,\left\langle A_{1}, A_{2}\right\rangle=-\frac{3}{2},\left\langle X_{j}, X_{j}\right\rangle=\frac{1}{4},\left\langle U_{j}, U_{j}\right\rangle=\frac{3}{4}$, and $(\mathfrak{a}+$ $\left.\mathfrak{n},\langle\rangle,, J_{1}, J_{2}, J_{3}\right)$ is a quaternion-Hermitian vector space.

\subsubsection{Homogeneous descriptions and structures of $A_{G_{2(2)}}$}

The four standard parabolic subalgebras of $\mathfrak{g}_{2(2)}$ are parametrized by the subsets $\Pi, \emptyset, \Psi_{1}=\left\{f_{1}-f_{2}\right\}$ and $\Psi_{2}=\left\{-f_{1}\right\}$ of $\Pi$. By using Theorems 2.2 and 2.3 we will obtain the homogeneous descriptions of $A_{G_{2(2)}}$.

The case $\Psi=\Pi$. We have $[\Psi]=\Sigma, \mathfrak{e}_{\Pi}^{\prime}=\mathfrak{a}_{\Pi}=\mathfrak{n}_{\Pi}=\{0\}$, and the refined Langlands decomposition is $\mathfrak{p}_{\Pi}=\mathfrak{g}_{2(2)}+\{0\}+\{0\}+\{0\}$. Here one only gets the transitive action of the full isometry group $G_{2(2)}$ on $A_{G_{2(2)}}$, and we have the description of $A_{G_{2(2)}}$ as the symmetric space $G_{2(2)} / S O(4)$. The associated reductive decomposition is the Cartan decomposition $\mathfrak{g}_{2(2)}=\mathfrak{k}+\mathfrak{p}$, with $\mathfrak{k} \cong$ $\mathfrak{s o}(4)$, and the corresponding structure is $S=0$.

The case $\Psi=\emptyset$. In this case, $\mathfrak{l}_{\emptyset}^{\prime}=\{0\}, \mathfrak{e}_{\emptyset}^{\prime}=Z_{\mathfrak{k}}(\mathfrak{a})=\{0\}$, and $\mathfrak{a}_{\emptyset}=$ $\mathfrak{a}$, and hence the refined Langlands decomposition of the minimal parabolic subalgebra is $\mathfrak{p}_{\emptyset}=\{0\}+\{0\}+\mathfrak{a}+\mathfrak{n}$. In this case, the only connected cocompact subgroup $\hat{G}$ which acts transitively on $A_{G_{2(2)}}$ is provided by $E=A$, so 
we have the description of $A_{G_{2(2)}}$ as the solvable Lie group $\hat{G}=A N$, where $N$ is the nilpotent factor in the Iwasawa decomposition of $G_{2(2)}$. The associated reductive decomposition is $\mathfrak{a}+\mathfrak{n}=\{0\}+(\mathfrak{a}+\mathfrak{n})$ and, having in mind that in this case $\mathfrak{m}=\mathfrak{a}+\mathfrak{n}$, by using (2.4) we obtain that the corresponding homogeneous quaternionic Kähler structure $S$ is given by Table III with $\delta=\varepsilon=1$.

\begin{tabular}{ccccccccc} 
Table III & $A_{1}$ & $A_{2}$ & $X_{1}$ & $X_{2}$ & $X_{3}$ & $U_{1}$ & $U_{2}$ & $U_{3}$ \\
\hline$S_{A_{1}}$ & 0 & 0 & 0 & 0 & 0 & 0 & 0 & 0 \\
$S_{A_{2}}$ & 0 & 0 & 0 & 0 & 0 & 0 & 0 & 0 \\
$S_{X_{1}}$ & $-\delta 2 X_{1}$ & $3 \delta X_{1}$ & $\frac{1}{2} \delta A_{1}$ & $\frac{1}{2} \delta X_{3}$ & $-\frac{1}{2} \delta X_{2}$ & 0 & $-\frac{1}{2} \delta U_{3}$ & $\frac{1}{2} \delta U_{2}$ \\
$S_{X_{2}}$ & $X_{2}$ & $-3 X_{2}$ & $-\frac{1}{2} X_{3}$ & $\frac{1}{2} A_{1}+\frac{1}{2} A_{2}$ & $\frac{1}{2} X_{1}$ & $\frac{1}{2} U_{3}$ & 0 & $-\frac{1}{2} U_{1}$ \\
$S_{X_{3}}$ & $-X_{3}$ & 0 & $-\frac{1}{2} X_{2}$ & $\frac{1}{2} X_{1}$ & $A_{1}+\frac{1}{2} A_{2}$ & $\frac{1}{2} U_{2}$ & $-\frac{1}{2} U_{1}$ & 0 \\
$S_{U_{1}}$ & 0 & $-U_{1}$ & 0 & $\frac{1}{2} U_{3}$ & $\frac{1}{2} U_{2}$ & $\frac{3}{2} A_{1}+A_{2}$ & $-\frac{3}{2} X_{3}-U_{3}$ & $-\frac{3}{2} X_{2}+U_{2}$ \\
$S_{U_{2}}$ & $-U_{2}$ & $U_{2}$ & $-\frac{1}{2} U_{3}$ & 0 & $-\frac{1}{2} U_{1}$ & $\frac{3}{2} X_{3}-U_{3}$ & $\frac{3}{2} A_{1}+\frac{1}{2} A_{2}$ & $\frac{3}{2} X_{1}+U_{1}$ \\
$S_{U_{3}}$ & $\varepsilon U_{3}$ & $-2 \varepsilon U_{3}$ & $-\frac{1}{2} \varepsilon U_{2}$ & $-\frac{1}{2} \varepsilon U_{1}$ & 0 & $\varepsilon\left(\frac{3}{2} X_{2}+U_{2}\right)$ & $\varepsilon\left(\frac{3}{2} X_{1}-U_{1}\right)$ & $\frac{1}{2} \varepsilon A_{2}$ \\
\hline
\end{tabular}

The case $\Psi=\Psi_{1}$. Then $\left[\Psi_{1}\right]=\left\{ \pm\left(f_{1}-f_{2}\right)\right\}$, and $\mathfrak{p}_{\Psi_{1}}=\mathfrak{l}_{\Psi_{1}}^{\prime}+\{0\}+$ $\mathfrak{a}_{\Psi_{1}}+\mathfrak{n}_{\Psi_{1}}$, where $\mathfrak{a}_{\Psi_{1}}=\left\langle 3 A_{1}+2 A_{2}\right\rangle, \mathfrak{n}_{\Psi_{1}}=\sum_{f \in \Sigma+, f \neq f_{1}-f_{2}} \mathfrak{g}_{f}=\left\langle X_{2}, X_{3}, U_{1}\right.$, $\left.U_{2}, U_{3}\right\rangle$, and

$$
\mathfrak{l}_{\Psi_{1}}^{\prime}=\left\{\left(\begin{array}{ccccccc}
0 & x & 0 & 0 & s & y & 0 \\
-x & 0 & 0 & 0 & y & -s & 0 \\
0 & 0 & 0 & 0 & 0 & 0 & 0 \\
0 & 0 & 0 & 0 & 0 & 0 & 0 \\
s & y & 0 & 0 & 0 & x & 0 \\
y & -s & 0 & 0 & -x & 0 & 0 \\
0 & 0 & 0 & 0 & 0 & 0 & 0
\end{array}\right)\right\}=\left\langle A_{1}, X_{1}, Y_{1}\right\rangle \cong \mathfrak{s l}(2, \mathbb{R}) .
$$

The connected Lie subgroup $E=A_{\Psi_{1}} \cong \mathbb{R}$ of $P_{\Psi_{1}}$ with Lie algebra $\mathfrak{a}_{\Psi_{1}}$ is the only possible choice to get a co-compact subgroup $\hat{G}=L E N_{\Psi_{1}}$ of $G_{2(2)}$, with $L=L_{\Psi_{1}}^{\prime} \cong S l(2, \mathbb{R})$, and $\hat{G}$ acts transitively on $A_{G_{2(2)}}$. The isotropy algebra is $\mathfrak{h}=\hat{\mathfrak{g}} \cap \mathfrak{k}=\mathfrak{l}_{\Psi_{1}}^{\prime} \cap \mathfrak{k}=\left\langle\left(X_{1}\right)_{\mathfrak{k}}\right\rangle \cong \mathfrak{s o}(2)$. We have the reductive decomposition $\hat{\mathfrak{g}}=\mathfrak{h}+\mathfrak{m}$, where $\hat{\mathfrak{g}}=\mathfrak{p}_{\Psi_{1}}$, and $\mathfrak{m}=\left\langle A_{1}, A_{2},\left(X_{1}\right)_{\mathfrak{p}}, X_{2}, X_{3}, U_{1}, U_{2}, U_{3}\right\rangle$, which is associated to the description $A_{G_{2(2)}} \equiv S l(2, \mathbb{R}) \mathbb{R} N_{\Psi_{1}} / S O(2)$. By using (2.4), we obtain that the corresponding homogeneous quaternionic Kähler structure $S$ is given at $o$ by Table III with $\delta=0, \varepsilon=1$.

The case $\Psi=\Psi_{2}$. Then $\left[\Psi_{2}\right]=\left\{ \pm f_{1}\right\}$ and $\mathfrak{p}_{\Psi_{2}}=\mathfrak{l}_{\Psi_{2}}^{\prime}+\{0\}+\mathfrak{a}_{\Psi_{2}}+\mathfrak{n}_{\Psi_{2}}$, where $\mathfrak{a}_{\Psi_{2}}=\left\langle 2 A_{1}+A_{2}\right\rangle, \mathfrak{n}_{\Psi_{2}}=\sum_{f \in \Sigma^{+}, f \neq-f_{1}} \mathfrak{g}_{f}=\left\langle X_{1}, X_{2}, X_{3}, U_{1}, U_{2}\right\rangle$, and

$$
\mathfrak{l}_{\Psi_{2}}^{\prime}=\left\{\left(\begin{array}{ccccccc}
0 & 0 & 0 & 2 u & -2 s & 0 & 0 \\
0 & 0 & u & 0 & 0 & s & v \\
0 & -u & 0 & 0 & 0 & -v & s \\
-2 u & 0 & 0 & 0 & 2 v & 0 & 0 \\
-2 s & 0 & 0 & 2 v & 0 & 0 & 0 \\
0 & s & -v & 0 & 0 & 0 & -u \\
0 & v & s & 0 & 0 & u & 0
\end{array}\right)\right\}=\left\langle A_{2}, U_{3}, V_{3}\right\rangle \cong \mathfrak{s l}(2, \mathbb{R}) .
$$

In this case we also have only one possible choice for $E$ as the connected subgroup $A_{\Psi_{2}} \cong \mathbb{R}$ of $P_{\Psi_{2}}$ whose Lie algebra is $\mathfrak{a}_{\Psi_{2}}$. So, we get a co-compact subgroup $\hat{G}=L E N_{\Psi_{2}}$ of $G_{2(2)}$, with $L=L_{\Psi_{2}}^{\prime} \cong S l(2, \mathbb{R})$, which acts transitively on $A_{G_{2(2)}}$. This gives $A_{G_{2(2)}} \equiv S l(2, \mathbb{R}) \mathbb{R} N_{\Psi_{2}} / S O(2)$, whose natural associated 
reductive decomposition is $\hat{\mathfrak{g}}=\mathfrak{h}+\mathfrak{m}$, where $\hat{\mathfrak{g}}=\mathfrak{p}_{\Psi_{2}}, \mathfrak{h}=\hat{\mathfrak{g}} \cap \mathfrak{k}=\mathfrak{l}_{\Psi_{2}}^{\prime} \cap \mathfrak{k}=$ $\left\langle\left(U_{3}\right)_{\mathfrak{k}}\right\rangle \cong \mathfrak{s o}(2)$ and $\mathfrak{m}=\left\langle A_{1}, A_{2}, X_{1}, X_{2}, X_{3}, U_{1}, U_{2},\left(U_{3}\right)_{\mathfrak{p}}\right\rangle$. By $(2.4)$, we have that the corresponding structure $S$ is given at $o$ by Table III with $\delta=1, \varepsilon=0$.

We have

Proposition 3.3. The space $A_{G_{2(2)}}$ admits the homogeneous quaternionic Kähler structures $S$ (associated to each corresponding homogeneous description $\hat{G} / H$ ) given by

\begin{tabular}{|l|l|}
\hline$S$ & $\hat{G} / H$ \\
\hline \hline 0 & $G_{2(2)} / S O(4)$ \\
Table III, $\delta=\varepsilon=1$ & $A N \quad\left(A \cong \mathbb{R}^{2}\right)$ \\
Table III, $\delta=0, \varepsilon=1$ & $S l(2, \mathbb{R}) \mathbb{R} N_{\Psi_{1}} / S O(2)$ \\
Table III, $\delta=1, \varepsilon=0$ & $S l(2, \mathbb{R}) \mathbb{R} N_{\Psi_{2}} / S O(2)$ \\
\hline
\end{tabular}

where $N$ is the nilpotent factor in the Iwasawa decomposition $G_{2(2)}=S O(4) A N$ and $\Psi_{1}$ and $\Psi_{2}$ are the subsets of simple restricted roots defining the intermediate parabolic subalgebras of $\left(\mathfrak{g}_{2(2)}, \mathfrak{a}\right)$.

\subsection{Types of homogeneous quaternionic Kähler structures}

For each parabolic subgroup $P_{\Psi}$ of the full connected isometry group $G$ of each non-compact quaternion-Kähler symmetric space $M$ of dimension 8, the subgroups $\hat{G}$ of $G$ acting transitively on $M$ are of the form $\hat{G}=L E N_{\Psi}$ as in Theorem 2.3, where $L=L_{\Psi}^{\prime}$. Moreover, $E$ is a connected closed subgroup of $E_{\Psi}^{\prime} A_{\Psi}$ such that the projection of its Lie algebra $\mathfrak{e} \subset \mathfrak{e}_{\Psi}^{\prime}+\mathfrak{a}_{\Psi}$ to $\mathfrak{a}_{\Psi}$ is surjective. If this projection is an isomorphism we say that $E$ is minimal. Then we have

Theorem 3.4. Let $G=K A N$ be the Iwasawa decomposition of each of the groups $S U(2,2), S p(2,1)$, and $G_{2(2)}$. The homogeneous descriptions of each eight-dimensional non-compact quaternion-Kähler symmetric space are of the form $\hat{G} / H$, where $\hat{G}=L_{\Psi}^{\prime} E N_{\Psi}$, with $L_{\Psi}^{\prime}$ either non-compact simple or trivial and $N_{\Psi}$ nilpotent. If $E$ is minimal, then it is simply-connected and abelian, and in this case the corresponding types of homogeneous quaternionic Kähler structures are given in the following table (where the figure on the fifth column, if any, stands for the number of parameters of the corresponding n-parametric family of homogeneous quaternionic Kähler structures).

\begin{tabular}{|l|l|l|l|c|l|l|}
\hline IV & $G / K$ & $\Psi$ & $L_{\Psi}^{\prime} E N_{\Psi} / H$ & $\operatorname{dim} E$ & $n$ & type \\
\hline \hline & $A_{S U(2,2)}$ & $\Pi$ & $S U(2,2) / S(U(2) \times U(2))$ & 0 & & $\{0\}$ \\
& & $\emptyset$ & $E_{\lambda, \mu} N \quad(\lambda, \mu \in \mathbb{R})$ & 2 & 2 & $\mathcal{Q} \mathcal{K}_{12345}$ \\
& & $\Psi_{1}$ & $S l(2, \mathbb{C}) A_{\Psi_{1}} N_{\Psi_{1}} / S U(2)$ & 1 & & $\mathcal{Q} \mathcal{K}_{135}$ \\
& & $\Psi_{2}$ & $S U(1,1) E_{\lambda} N_{\Psi_{2}} / U(1)$ & 1 & 1 & $\mathcal{Q} \mathcal{K}_{12345}$ \\
\hline \hline & $A_{S p(2,1)}$ & $\Pi$ & $S p(2,1) /(S p(2) \times S p(1))$ & 0 & & $\{0\}$ \\
& $\emptyset$ & $E_{\lambda, \mu} N \quad\left(\lambda, \mu \in \mathbb{R}^{3} \backslash\{0\}\right)$ & 1 & 6 & $\mathcal{Q} \mathcal{K}_{12345}$ \\
& & $\emptyset$ & $E_{0, \mu} N \quad\left(\mu \in \mathbb{R}^{3} \backslash\{0\}\right)$ & 1 & 3 & $\mathcal{Q} \mathcal{K}_{1345}$ \\
& & $\emptyset$ & $A N=E_{0,0} N$ & 1 & & $\mathcal{Q \mathcal { K }}{ }_{134}$ \\
\hline \hline & $A_{G_{2(2)}}$ & $\Pi$ & $G_{2(2)} / S O(4)$ & 0 & & $\{0\}$ \\
& & $\emptyset$ & $A N$ & 2 & & $\mathcal{Q} \mathcal{K}_{12345}$ \\
& & $\Psi_{1}$ & $S l(2, \mathbb{R}) A_{\Psi_{1}} N_{\Psi_{1}} / S O(2)$ & 1 & & $\mathcal{Q} \mathcal{K}_{12345}$ \\
& & $\Psi_{2}$ & $S l(2, \mathbb{R}) A_{\Psi_{2}} N_{\Psi_{2}} / S O(2)$ & 1 & & $\mathcal{Q \mathcal { K }} \mathcal{K}_{12345}$ \\
\hline
\end{tabular}


Proof. In order to determine the types of non-zero homogeneous quaternionic Kähler structures (see Theorem 1.3) on the three spaces, we obtain for each structure the forms $\theta^{i}$ satisfying equations (1.3), by using the formula (2.6).

Case 1. The space $A_{S U(2,2)}$. The values of the forms $\theta^{i}$ at $o \in A_{S U(2,2)}$ corresponding to the homogeneous quaternionic Kähler structure $S$ given in Table I are as follows

\begin{tabular}{ccccccccc} 
& $A_{1}$ & $A_{2}$ & $U_{1}$ & $U_{2}$ & $X_{1}$ & $X_{1}^{\prime}$ & $X_{2}$ & $X_{2}^{\prime}$ \\
\hline$\theta^{1}$ & $2 \lambda$ & $2 \mu$ & 1 & $-\delta$ & 0 & 0 & 0 & 0 \\
$\theta^{2}$ & 0 & 0 & 0 & 0 & -2 & 0 & $2 \varepsilon$ & 0 \\
$\theta^{3}$ & 0 & 0 & 0 & 0 & 0 & -2 & 0 & $2 \varepsilon$ \\
\hline
\end{tabular}

If $\Psi=\emptyset,(\delta=\varepsilon=1)$, the structure $S=S^{\lambda, \mu}(\lambda, \mu \in \mathbb{R})$ decomposes as $S=\Theta+T$, with $\Theta \in \mathcal{Q K}_{12}$ (i.e., $\Theta_{X} Y=\frac{1}{2} \sum_{a=1}^{3} \theta^{a}(X) J_{a} Y$ ), and $T \in \mathcal{Q} \mathcal{K}_{345}$. The condition for the tensor $\Theta$ to be in $\mathcal{Q} \mathcal{K}_{1}$ is $\theta^{a}=\theta \circ J_{a}, a=1,2,3$, for some 1form $\theta$; and this condition is not satisfied. In turn, $\Theta \in \mathcal{Q} \mathcal{K}_{2}$ if $\sum_{a=1}^{3} \theta^{a} \circ J_{a}=0$, which is not satisfied for instance by $A_{1}$. Hence $\Theta \in \mathcal{Q} \mathcal{K}_{12} \backslash\left(\mathcal{Q} \mathcal{K}_{1} \cup \mathcal{Q} \mathcal{K}_{2}\right)$. The relevant trace of $T$ is $c_{12}(T)=\left\langle\frac{7}{2} A_{1}+\frac{3}{2} A_{2}+\lambda U_{1}-\mu U_{2}, \cdot\right\rangle$. On the other hand, since $\operatorname{dim} M=8$, the form defining the $\mathcal{Q} \mathcal{K}_{3}$-component of $T$ is given by $\vartheta=\frac{1}{10} c_{12}$. Hence $\vartheta=\frac{1}{20}\left\langle 7 A_{1}+3 A_{2}+2 \lambda U_{1}-2 \mu U_{2}, \cdot\right\rangle$, so the $\mathcal{Q K}_{3^{-}}$ component of $T$ does not vanish either. Consider now the operator $F: \hat{\mathcal{V}} \rightarrow \hat{\mathcal{V}}$ defined by $F(T)_{X Y Z}=T_{Y Z X}-T_{Z Y X}+\sum_{a=1}^{3}\left(T_{J_{a} Y J_{a} Z X}-T_{J_{a} Z J_{a} Y X}\right)$, with eigenvalues 2 and -4 and respective eigenspaces $\mathcal{Q} \mathcal{K}_{34}$ and $\mathcal{Q} \mathcal{K}_{5}$ (see Theorem 1.3). Take the tensor in $\mathcal{Q} \mathcal{K}_{3}$ given by $T_{X Y Z}^{\vartheta}=\langle X, Y\rangle \vartheta(Z)-\langle X, Z\rangle \vartheta(Y)+$ $\sum_{a=1}^{3}\left(\left\langle X, J_{a} Y\right\rangle \vartheta\left(J_{a} Z\right)-\left\langle X, J_{a} Z\right\rangle \vartheta\left(J_{a} Y\right)\right)$. Then $T-T^{\vartheta} \in \mathcal{Q} \mathcal{K}_{45}$ and we get $F\left(T-T^{\vartheta}\right)_{X Y Z}=F(T)_{X Y Z}-2 T_{X Y Z}^{\vartheta}$. In particular, we obtain $\left(T-T^{\vartheta}\right)_{U_{2} X_{1}^{\prime} X_{2}}=$ $-\frac{17}{10}$ and $F\left(T-T^{\vartheta}\right)_{U_{2} X_{1}^{\prime} X_{2}}=\frac{18}{5}$, so $T-T^{\vartheta} \in \mathcal{Q} \mathcal{K}_{45} \backslash\left(\mathcal{Q} \mathcal{K}_{4} \cup \mathcal{Q} \mathcal{K}_{5}\right)$. Hence the tensor $S$ has a non-zero component in each subspace $\mathcal{Q} \mathcal{K}_{j}, j=1, \ldots, 5$.

If $\Psi=\Psi_{1},(\lambda=\mu=\varepsilon=0, \delta=1)$, we have $S=\Theta+T$, where $\Theta \in$ $\mathcal{Q} \mathcal{K}_{1}$, with corresponding 1 -form $\theta=\left\langle A_{1}+A_{2}, \cdot\right\rangle$, and $T$ having non-zero $\mathcal{Q K}_{3^{-}}$ component, with corresponding 1-form $\vartheta=\frac{1}{4}\left\langle A_{1}+A_{2}, \cdot\right\rangle$. Moreover, we get $\left(T-T^{\vartheta}\right)_{U_{1} X_{2}^{\prime} X_{1}}=-\frac{3}{2}$ and $F\left(T-T^{\vartheta}\right)_{U_{1} X_{2}^{\prime} X_{1}}=6=-4\left(T-T^{\vartheta}\right)_{U_{1} X_{2}^{\prime} X_{1}}$. So $T-T^{\vartheta} \notin \mathcal{Q} \mathcal{K}_{4}$. As a computation with Maple shows, this happens for any choice of vectors. Hence $S \in \mathcal{Q} \mathcal{K}_{135}$.

If $\Psi=\Psi_{2},(\mu=\delta=0, \varepsilon=1)$, then $S=S^{\lambda}(\lambda \in \mathbb{R})$ decomposes as $S=\Theta+T$ with $\Theta \in \mathcal{Q} \mathcal{K}_{12} \backslash\left(\mathcal{Q} \mathcal{K}_{1} \cup \mathcal{Q} \mathcal{K}_{2}\right)$. Since moreover $\vartheta=\frac{1}{20}\left\langle 7 A_{1}+2 \lambda U_{1}, \cdot\right\rangle$, $\left(T-T^{\vartheta}\right)_{X_{1}^{\prime} U_{2} X_{2}}=-\frac{13}{20}$, and $F\left(T-T^{\vartheta}\right)_{X_{1}^{\prime} U_{2} X_{2}}=\frac{6}{5}$, the tensor $S$ has a non-zero component in each subspace $\mathcal{Q} \mathcal{K}_{j}, j=1, \ldots, 5$.

Case 2. The space $A_{S p(2,1)}$. The forms $\theta^{i}$ corresponding to the homogeneous quaternionic Kähler structure $S=S^{\lambda, \mu}\left(\lambda, \mu \in \mathbb{R}^{3}\right)$ given in Table II have the following values at $o \in A_{S p(2,1)}$

\begin{tabular}{rrrrrrrrr} 
& $A_{0}$ & $X_{1}$ & $U_{1}$ & $U_{1}^{\prime}$ & $X_{2}$ & $X_{2}^{\prime}$ & $U_{2}$ & $U_{2}^{\prime}$ \\
\hline$\theta^{1}$ & $2 \lambda_{1}$ & 2 & 0 & 0 & 0 & 0 & 0 & 0 \\
$\theta^{2}$ & $-2 \lambda_{2}$ & 0 & 2 & 0 & 0 & 0 & 0 & 0 \\
$\theta^{3}$ & $-2 \lambda_{3}$ & 0 & 0 & 2 & 0 & 0 & 0 & 0 \\
\hline
\end{tabular}


Equations (1.4) are satisfied, with the following nonzero values of $\theta^{i}$ at $o$ : $\theta^{1}\left(A_{0}\right)=2 \lambda_{1}, \theta^{2}\left(A_{0}\right)=-2 \lambda_{2}, \theta^{3}\left(A_{0}\right)=-2 \lambda_{3}, \theta^{1}\left(X_{1}\right)=\theta^{2}\left(U_{1}\right)=\theta^{3}\left(U_{1}^{\prime}\right)=2$. We have $S=\Theta+T$, with $\Theta \in \mathcal{Q K}_{12} \backslash \mathcal{Q} \mathcal{K}_{1} \cup \mathcal{Q} \mathcal{K}_{2}$, except for $\lambda_{1}=\lambda_{2}=\lambda_{3}=0$. In this case $\Theta \in \mathcal{Q} \mathcal{K}_{1}$, with corresponding 1-form $\theta=2\left\langle A_{0}, \cdot\right\rangle$. As for the specific type of $T$ inside $\mathcal{Q} \mathcal{K}_{345}$, we first have that $\vartheta=\frac{1}{10}\left\langle 7 A_{0}+\lambda_{1} X_{1}-\lambda_{2} U_{1}-\right.$ $\left.\lambda_{3} U_{1}^{\prime}, \cdot\right\rangle$, so the $\mathcal{Q} \mathcal{K}_{3}$-component of $T$ does not vanish. To find the $\mathcal{Q K}_{45^{-}}$ component, we have for example $\left(T-T^{\vartheta}\right)_{U_{2}^{\prime} X_{2} U_{1}^{\prime}}=-\frac{3}{10}$ and $F\left(T-T^{\vartheta}\right)_{U_{2}^{\prime} X_{2} U_{1}^{\prime}}=$ $-\frac{3}{5}$, so $T-T^{\vartheta}$ is never in $\mathcal{Q K}_{5}$. Suppose now that at least one of the parameters $\lambda_{i}, \mu_{i}, i=1,2,3$, is non-zero. Computing now as before, we get for instance for $\mu_{2} \neq 0$ that $(T-T)_{A_{0} X_{2} U_{2}}=-\mu_{2}$ and $F\left(T-T^{\vartheta}\right)_{A_{0} X_{2} U_{2}}=2 \mu_{2}$, which is not equal to $2\left(T-T^{\vartheta}\right)_{A_{0} X_{2} U_{2}}$. This also happens for the other parameters. Hence $T \in \mathcal{Q K} \mathcal{K}_{345}$. If finally $\lambda_{i}, \mu_{i}, i=1,2,3$, are all zero, a computation with Maple shows that $T \in \mathcal{Q K}_{34}$ (see also [6, Prop. 5.3]).

Case 3. The space $A_{G_{2(2)}}$. The values of the forms $\theta^{i}$ at $o \in A_{G_{2(2)}}$ corresponding to the homogeneous quaternionic Kähler structure $S$ given in Table III are the following

\begin{tabular}{ccccccccc} 
& $A_{1}$ & $A_{2}$ & $X_{1}$ & $X_{2}$ & $X_{3}$ & $U_{1}$ & $U_{2}$ & $U_{3}$ \\
\hline$\theta^{1}$ & 0 & 0 & $\frac{\delta}{2}$ & 0 & 0 & $-\frac{3}{2}$ & 0 & 0 \\
$\theta^{2}$ & 0 & 0 & 0 & $\frac{1}{2}$ & 0 & 0 & $\frac{3}{2}$ & 0 \\
$\theta^{3}$ & 0 & 0 & 0 & 0 & $\frac{1}{2}$ & 0 & 0 & $-\frac{3 \varepsilon}{2}$ \\
\hline
\end{tabular}

In any of the three cases, that is, $\Psi=\emptyset,(\delta=\varepsilon=1), \Psi=\Psi_{1},(\delta=0, \varepsilon=1)$, and $\Psi=\Psi_{2},(\delta=1, \varepsilon=0)$, we have $S=\Theta+T$, with $\Theta \in \mathcal{Q} \mathcal{K}_{12} \backslash\left(\mathcal{Q K}_{1} \cup \mathcal{Q K} \mathcal{K}_{2}\right)$. Since moreover one has

\begin{tabular}{lllll}
$\Psi$ & $\vartheta$ & $\left(T-T^{\vartheta}\right)_{X Y Z}$ & $F\left(T-T^{\vartheta}\right)_{X Y Z}$ & vectors $X Y Z$ \\
\hline$\emptyset$ & $\frac{1}{30}\left\langle 21 A_{1}+11 A_{2}, \cdot\right\rangle$ & $\frac{1}{20}$ & $-\frac{29}{160}$ & $X_{1} X_{2} X_{3}$ \\
$\Psi_{1}$ & $\frac{11}{60}\left\langle 3 A_{1}+2 A_{2}, \cdot\right\rangle$ & $\frac{49}{160}$ & $-\frac{29}{80}$ & $X_{2} X_{3} X_{1}$ \\
$\Psi_{2}$ & $\frac{7}{20}\left\langle 2 A_{1}+A_{2}, \cdot\right\rangle$ & $\frac{1}{20}$ & $-\frac{73}{320}$ & $X_{1} X_{2} X_{3}$ \\
\hline
\end{tabular}

it follows that the tensor $S$ has a non-zero component in each primitive subspace $\mathcal{Q K}_{j}, j=1, \ldots, 5$.

\section{References}

[1] D. V. Alekseevskiı̌, Classification of quaternionic spaces with a transitive solvable group of motions, Math. USSR Izvestija 9 (1975), 297-339.

[2] D. V. Alekseevsky and V. Cortés, Homogeneous quaternionic Kähler manifolds of unimodular groups, Boll. Un. Mat. Ital. B (7) 11 (1997), no. 2, suppl., 217-229.

[3] W. Ambrose and I. M. Singer, On homogeneous Riemannian manifolds, Duke Math. J. 25 (1958), 647-669.

[4] J. Bagger and W. Witten, Matter couplings in $N=2$ supergravity, Nucl. Phys. B 222 (1983), 1-10.

[5] M. Castrillón López, P. M. Gadea, and J. A. Oubiña, Homogeneous quaternionic Kähler structures on 12-dimensional Alekseevsky spaces, J. Geom. Phys. 57 (2007), doi:10.1016/j.geomphys.2007.05.007. 
[6] M. Castrillón López, P. M. Gadea, and A. F. Swann, Homogeneous quaternionic Kähler structures and quaternionic hyperbolic space, Transform. Groups 11 (2006), 575-608.

[7] S. Cecotti, Homogeneous Kähler manifolds and T-algebras in $N=2$ supergravity and superstrings, Comm. Math. Phys. 124 (1989), 23-55.

[8] V. Cortés, Alekseevskian spaces, Diff. Geom. Appl. 6 (1996), 129-168.

[9] A. Fino, Intrinsic torsion and weak holonomy, Math. J. Toyama Univ. 21 (1998), $1-22$.

[10] P. Fré, F. Gargiulo, J. Rosseel, K. Rulik, M. Trigiante, and A. Van Proeyen, TitsSatake projections of homogeneous special geometries, Classical Quantum Gravity 24 (2007), 27-78.

[11] P. M. Gadea and J. A. Oubiña, Reductive homogeneous pseudo-Riemannian manifolds, Monatsh. Math. 124 (1997), 17-34.

[12] C. S. Gordon and E. N. Wilson, Isometry groups of Riemannian solvmanifolds, Trans. Amer. Math. Soc. 307 (1988), 245-269.

[13] J. Heber, Noncompact homogeneous Einstein spaces, Invent. Math. 133 (1998), 279-352.

[14] V. F. Kiričenko, On homogeneous Riemannian spaces with an invariant structure tensor, Dokl. Akad. Nauk SSSR 252 (1980), 291-293.

[15] S. Kobayashi and K. Nomizu, Foundations of differential geometry. Volume II, Tracts in Mathematics, Number 15, Wiley, New York, 1969.

[16] P. Meessen, Homogeneous Lorentzian spaces admitting a homogeneous structure of type $\mathcal{T}_{1} \oplus \mathcal{T}_{3}$, J. Geom. Phys. 56 (2006), 754-761.

[17] A. Montesinos Amilibia, Degenerate homogeneous structures of type $\mathcal{S}_{1}$ on pseudoRiemannian manifolds, Rocky Mountain J. Math. 31 (2001), 561-579.

[18] F. Tricerri and L. Vanhecke, Homogeneous structures on Riemannian Manifolds, London Math. Soc. Lect. Notes Ser. 83, Cambridge Univ. Press, Cambridge, U.K., 1983.

[19] B. de Wit and A. Van Proeyen, Special geometry, cubic polynomials and homogeneous quaternionic spaces, Comm. Math. Phys. 149 (1992), 307-333.

[20] D. Witte Morris, Cocompact subgroups of semisimple Lie groups, Lie algebras and related topics, Proc. Res. Conf., Madison, WI, 1988, Contemp. Math. 110 (1990), 309-313.

[21] J. A. Wolf, Complex homogeneous contact manifolds and quaternionic symmetric spaces, J. Math. Mech. 14 (1965), 1033-1047.

\section{Authors' addresses:}

M. C. L.: Departamento de Geometría y Topología, Facultad de Matemáticas, Av. Complutense s/n, 28040-Madrid, Spain. E-mail: mcastri@mat.ucm.es

P. M. G.: Institute of Mathematics and Fundamental Physics, CSIC, Serrano 123, 28006-Madrid, Spain. E-mail: pmgadea@iec.csic.es

J. A. O.: Departamento de Xeometría e Topoloxía, Facultade de Matemáticas, Universidade de Santiago de Compostela, 15782-Santiago de Compostela, Spain. E-mail: jaoubina@usc.es 